\title{
Sparse and Switching Infinite Horizon Optimal Controls with Mixed-Norm Penalizations
}

\author{
Dante Kalise* $\quad$ Karl Kunisch $^{\dagger} \quad$ Zhiping Rao $^{\ddagger}$
}

\begin{abstract}
A class of infinite horizon optimal control problems involving mixed quasi-norms of $L^{p}$-type cost functionals for the controls is discussed. These functionals enhance sparsity and switching properties of the optimal controls. The existence of optimal controls and their structural properties are analyzed on the basis of first order optimality conditions. A dynamic programming approach is used for numerical realization.
\end{abstract}

Keywords: Optimal control, infinite horizon control, sparse controls, switching controls, optimality conditions, dynamic programming.

AMS Classification: 93D15, 93B52, 93C05, 93C20

\section{Introduction}

In this work we continue our investigations of infinite horizon optimal control problems with nonconvex cost functionals which we started in [23]. We focus on optimal control of nonlinear dynamical systems which are affine in the control. The input control is a vector-valued function $u=\left(u_{1}, \ldots, u_{m}\right)$ in the space $L^{\infty}\left(0, \infty ; \mathbb{R}^{m}\right)$ under control constraints. The focus rests on that part of the cost functional which involves the control. It is given as follows:

$$
\int_{0}^{\infty}\left(\sum_{i=1}^{m}\left|u_{i}(t)\right|^{p}\right)^{q / p} d t
$$

where $0<p<1$ and $p \leq q \leq 1$. This functional is nonsmooth and nonconvex, leading to a challenging optimal control problem with interesting properties for the optimal control laws, in particular sparsity and switching. It appears that the terminology "sparse" is not rigorously defined in the literature, but generally it is used to describe the property of the optimal control to be identically zero over nontrivial subsets of the temporal domain. Here, by sparsity we refer to the situation in which the whole vector $u(t)$ is zero. Switching control, is related to coordinate-wise sparsity, and is used to describe the property

$$
u_{i}(t) u_{j}(t)=0 \text { for } i, j \in\{1, \ldots, m\}, i \neq j, t \geq 0,
$$

\footnotetext{
${ }^{*}$ School of Mathematical Sciences, University of Nottingham, University Park, Nottingham NG7 2QL, United Kingdom. Email: dante.kalise@ nottingham.ac.uk . Research supported by the Imperial College London Research Fellowship program.

${ }^{\dagger}$ Institute for Mathematics and Scientific Computing, University of Graz, Heinrichstraße 36, A-8010 Graz, Austria, and Radon Institute of Computational and Applied Mathematics, Austrian Academy of Sciences. Email: karl.kunisch@unigraz.at. Research in part supported by the ERC advanced grant 668998 (OCLOC) under the EU's H2020 research program.

${ }^{\ddagger}$ School of Mathematics and Statistics, and Hubei Key Laboratory of Computational Sciences, Wuhan University, Wuhan, 430072, Hubei, People's Republic of China. Email: zhiping.rao@whu.edu.cn. Research in part supported by NSFC 11801422 .
} 
which is equivalent to saying that at most one coordinate of $u(t)$ is non-zero at $t$. While the use of the control penalty (1.1) does not guarantee sparsity nor switching properties, it enhances them. This is illustrated in Figure 1, where unit balls for different $q / p$ ratios are shown. For a fixed $q$ decreasing $p$ (column-wise in the sub-figure) one direction becomes dominant over the other.
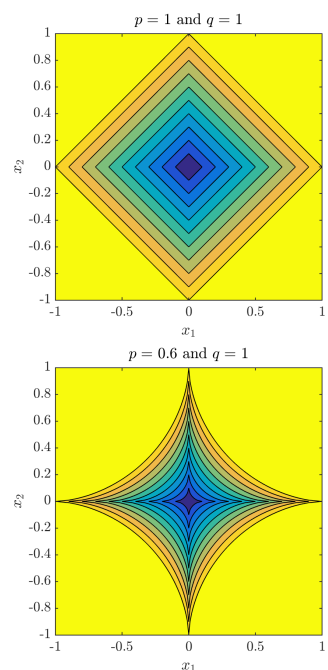

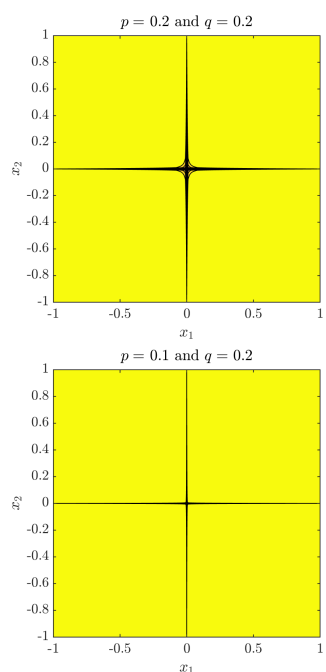

Figure 1: Contour levels (0.1 to 1$)$ of different balls $\|u\|_{p}^{q}$.

To further illustrate the effect of (1.1) let us consider the case $p=1 / 2$ and $q=1$. Then the running cost for the control is given by

$$
\sum_{i=1}^{m}\left|u_{i}(t)\right|+2 \sum_{i, j \in\{1, \ldots, m\}, i \neq j}\left|u_{i}(t) u_{j}(t)\right|^{1 / 2}
$$

where the $L^{1}$-penalization on $u_{i}$ will support sparsity in the control and the product penalization enhances switching phenomena. More generally, if $\frac{q}{p}=j \in \mathbb{N}$ is an integer, then the running cost is is combination of an $L^{q}$-penalization on each control coordinate $u_{i}$, and it further contains weighted summands of (up to) $j$ - tuples of fractional powers of $\left|u_{i}\right|$, with the sum of the powers for each tuple summing to $q$. Fixing $q$, and decreasing $p$ we expect that the control cost $(1.1)$ increases the switching nature of the optimal controls, since the weights on the tuples compared to those on the singletons increase. Moreover, decreasing $q$ we expect that the subdomain over which the optimal control vanishes (in all coordinates) increases. These properties will be illustrated by numerical experiments.

The case with $p=q$ and $0<p \leq 1$ has been studied in [23]. Existence and sparsity properties of optimal controls have been analyzed for this case, and these properties have been observed in the numerical simulations in the case with $0<p=q<1$. In the present work, the analysis is made for more general nonconvex problems with the control cost (1.1). Concerning the question of existence of optimal solutions, which is not guaranteed in general, we follow the ideas from [23] to reformulate the problem in infinite-dimensional sequence spaces by descretizing the controls, and extending an important result on weakly sequentially continuous mappings from [21] to obtain the existence result for our purposes.

The analysis of the sparsity and switching structure is based on optimality conditions. For this purpose we derive the necessary first order optimality conditions of the original problem, which follow from general results which are available in the literature. We also derive sufficient optimality 
condition for the reformulated problems. Subsequently, we investigate the sparsity and switching properties of the optimal controls under box constraints. Finally, by using dynamic programming techniques, optimal control laws are approximated globally in the state space for linear and nonlinear dynamical systems.

Let us mention previous related work on sparse and switching control. Closed-loop infinite horizon sparse optimal control problems with $L^{p}(0<p \leq 1)$ functionals were analyzed in [23]. Open-loop, finite horizon $L^{1}$ sparse optimal control for dynamical systems have been studied in e.g. [16, 28, 3]. Open-loop, finite horizon sparse optimal control for partial differential equations was studied in e.g. [20, 8, 25]. The Hamilton-Jacobi-Bellman equation for impulse and switching controls was discussed in [6, 30]. The synthesis of sparse feedback laws via dynamic programming has been studied in [14, 22, 1]. In the context of partial differential equations optimal control of systems switching among different modes were analysed in [18, 19], problems with convex switching enhancing functionals were investigated in [13, 11], and problems with nonconvex switching penalization in [12]. In [31] switching controls based on functionals suggested by controllability considerations were investigated. Mixed (quasi-)norms as in 1.1 with $p \neq q$ have been used earlier, though typically in convex situations with $p \geq 1, q \geq 1$. These investigations were carried out in the context of machine learning, regression analysis, and mathematical imaging, with the goal of achieving group sparsity or structured parsimony, see e.g. [6, 15, 24, 29, 32], and the references given there.

The structure of the paper is the following. The short section 2 contains the precise problem formulation. Existence of optimal controls, which are discretized in time, is obtained in section 3. The sparsity and switching structure of the optimal controls is analyzed on the basis of the optimality conditions for the time-continuous as well as the time discrete problems in sections 4 and 5 , respectively, and section 6 contains numerical results.

\section{Optimal control problem}

Let $U \subset \mathbb{R}^{m}$ be a closed set and let $f_{i}: \mathbb{R}^{d} \rightarrow \mathbb{R}^{d}$ be continuous differentiable functions for $i=$ $0, \ldots, m$. We consider the following control system: given $x \in \mathbb{R}^{d}$,

$$
\left\{\begin{array}{l}
\left.\dot{y}(t)=f_{0}(y(t))+\sum_{i=1}^{m} f_{i}(y(t)) u_{i}(t) \text { in }\right] 0, \infty[ \\
y(0)=x
\end{array}\right.
$$

Here $y(t) \in \mathbb{R}^{d}$ is the state variable and $u(t)=\left(u_{1}(t), \ldots, u_{m}(t)\right) \in \mathbb{R}^{m}$ is the input control. Given $p \in] 0,1\left[\right.$, we set for the vector $u=\left(u_{1}, \ldots, u_{m}\right) \in \mathbb{R}^{m}$

$$
\|u\|_{p}=\left(\sum_{i=1}^{m}\left|u_{i}\right|^{p}\right)^{1 / p}
$$

Let $q \in[p, 1], \lambda>0, \gamma>0$ and $y_{d} \in \mathbb{R}^{d}$. For any $x \in \mathbb{R}^{d}$, consider the cost functional

$$
J(x, u):=\int_{0}^{\infty} e^{-\lambda t}\left(\frac{1}{2}\left\|y(t)-y_{d}\right\|_{2}^{2}+\gamma\|u(t)\|_{p}^{q}\right) d t
$$

where $(y, u)$ satisfies the state equation 2.2), and the infinite horizon optimal control problem

$$
\inf \left\{J(x, u): u \in L^{\infty}(0, \infty ; U)\right\} .
$$

In (2.3), $\lambda$ is called the discount factor, $\gamma$ is the weight of control cost and $\|\cdot\|_{2}$ is the Euclidean norm in $\mathbb{R}^{d}$. The following assumptions are made. 
(H1) The control set $U$ is compact and convex.

(H2) There exists $L>0$ such that $\left\|f_{i}\left(x_{1}\right)-f_{i}\left(x_{2}\right)\right\|_{2} \leq L\left\|x_{1}-x_{2}\right\|_{2}$ for all $x_{1}, x_{2} \in \mathbb{R}^{d}$, and $i=$ $0, \ldots, m$.

(H3) For each $x \in \mathbb{R}^{d}$, there exists $u \in L^{\infty}(0, \infty ; U)$ such that $J(x, u)<\infty$.

Let us mention that the cost functional $J$ is convex in the state variable and nonconvex in the control. The case $q=p$ has been discussed in [23].

\section{Time-discretized model}

Since the cost functional $J$ is not convex in $u$, existence of optimal controllers for problem 2.4 does not hold in general. For this purpose we analyze the existence in the case of a time-discretized approximation to (2.4). We introduce the temporal grid $\left(t_{k}\right)_{k \in \mathbb{N}}$ :

$$
0=t_{0}<t_{1}<\cdots<t_{k}<t_{k+1}<\cdots,
$$

and denote by $I_{k}=\left[t_{k}, t_{k+1}[\right.$ for $k \in \mathbb{N}$. The control is then restricted to the following set of piecewise constant functions:

$$
U^{\Delta}=\left\{u=\left(u_{1}, \ldots, u_{m}\right) \in L^{\infty}(0, \infty ; U): u_{i}(t)=u_{i, k} \text { for } t \in I_{k}, i=1, \ldots, m, k \in \mathbb{N}\right\} .
$$

Consider the following optimal control problem

$$
\inf _{u \in U^{\Delta}} J^{\Delta}(x, u):=\int_{0}^{\infty} e^{-\lambda t}\left(\frac{1}{2}\left\|y(t)-y_{d}\right\|_{2}^{2}+\gamma\left(\sum_{i=1}^{m} \sum_{k=0}^{\infty}\left|u_{i, k}\right|^{p} \mathbb{1}_{I_{k}}(t)\right)^{q / p}\right) d t,
$$

where $y$ solves 2.2. A direct computation shows that

$$
J^{\Delta}(x, u)=\int_{0}^{\infty} e^{-\lambda t} \frac{1}{2}\left\|y(t)-y_{d}\right\|_{2}^{2} d t+\gamma \sum_{k=0}^{\infty} b_{k}\left(\sum_{i=1}^{m}\left|u_{i, k}\right|^{p}\right)^{q / p},
$$

where

$$
b_{k}=\int_{I_{k}} e^{-\lambda t} d t=\frac{1}{\lambda}\left(e^{-\lambda t_{k}}-e^{-\lambda t_{k+1}}\right) .
$$

For any $r>0$, the infinite dimensional sequence space $\ell^{r}=\left\{u \in \ell^{\infty}: \sum_{k=1}^{\infty}\left|u_{k}\right|^{r}<\infty\right\}$ is endowed with

$$
\|u\|_{r}=\left(\sum_{k=0}^{\infty}\left|u_{k}\right|^{r}\right)^{1 / r} .
$$

For convenience we recall that $\ell^{r}$, with $1<r<\infty$, are reflexive Banach spaces and $\ell^{r_{1}} \subset \ell^{r_{2}}$ if $1 \leq r_{1}<r_{2} \leq \infty$. To investigate the existence of optimal controls, we follow the idea introduced in [21] by defining the following reparametrization. For $\left\{b_{k}\right\}_{k=1}^{\infty}$ as above we define $\ell_{b}^{q}\left(\ell^{p}\right)$ as the space of all $\mathbb{R}^{m}$-valued sequences $u$ such that $\sum_{k=0}^{\infty} b_{k}\left(\sum_{i=1}^{m}\left|u_{i, k}\right|^{p}\right)^{q / p}<\infty$. In particular $u_{i, k}$ denotes the i-th coordinate of the k-th sequence element $u_{, k}$. Similarly $\ell^{q / p}\left(\ell^{1}\right)$ stands for the space of all $\mathbb{R}^{m}$-valued sequences $w$ such that $\sum_{k=0}^{\infty}\left(\sum_{i=1}^{m}\left|w_{i, k}\right|\right)^{q / p}<\infty$. We introduce the mapping $\Phi: \ell^{q / p}\left(\ell^{1}\right) \rightarrow \ell_{b}^{q}\left(\ell^{p}\right)$ by

$$
(\Phi(w))_{i, k}=\phi\left(w_{i, k}\right), \text { where } \phi\left(w_{i, k}\right)=b_{k}^{-1 / q}\left|w_{i, k}\right|^{1 / p} \operatorname{sgn}\left(w_{i, k}\right) .
$$

We have the following lemma: 
Lemma 3.1. The mapping $\Phi: \ell^{q / p}\left(\ell^{1}\right) \rightarrow l_{b}^{q}\left(\ell^{P}\right)$ is an isomorphism.

Proof. For $w \in \ell^{q / p}\left(\ell^{1}\right)$ we have

$$
\begin{aligned}
\|\Phi(w)\|_{\ell_{b}^{q}\left(\ell^{p}\right)} & =\left(\sum_{k=0}^{\infty} b_{k}\left(\sum_{i=1}^{m}\left|\phi\left(w_{i, k}\right)\right|^{p}\right)^{q / p}\right)^{1 / q}=\left(\sum_{k=0}^{\infty} b_{k}\left(\sum_{i=1}^{m} b_{k}^{-p / q}\left|w_{i, k}\right|\right)^{q / p}\right)^{1 / q} \\
& =\left(\sum_{k=0}^{\infty}\left(\sum_{i=1}^{m}\left|w_{i, k}\right|\right)^{q / p}\right)^{1 / q}=\|w\|_{\ell q / p\left(\ell^{\prime}\right)}^{1 / p}<\infty,
\end{aligned}
$$

and thus $\Phi(w) \in \ell_{b}^{q}\left(\ell^{p}\right)$ as desired. The inverse to $\Phi$ is given by

$$
\left(\Phi^{-1}(u)\right)_{i, k}=b_{k}^{p / q}\left|u_{i, k}\right|^{p} \operatorname{sgn}\left(u_{i, k}\right)
$$

and this concludes the proof.

From Lemma 3.1 and 3.6 it follows that 3.5 is equivalent to

$$
\inf _{w:(\Phi(w))_{, k} \in U} \frac{1}{2} \int_{0}^{\infty} e^{-\lambda t}\left\|y(t)-y_{d}\right\|_{2}^{2} d t+\gamma \sum_{k=0}^{\infty}\left(\sum_{i=1}^{m}\left|w_{i, k}\right|\right)^{q / p}
$$

where $y(\cdot)$ satisfies

$$
\left\{\begin{array}{l}
\dot{y}(t)=f_{0}(y(t))+\sum_{i=1}^{m} f_{i}(y(t)) b_{k}^{-1 / q}\left|w_{i, k}\right|^{1 / p} \operatorname{sgn}\left(w_{i, k}\right) \text { for } t \in\left[t_{k}, t_{k+1}\right), k=0,1, \ldots \\
y(0)=x
\end{array}\right.
$$

Thus the relationship between the controls on $\left[t_{k}, t_{k+1}\right)$ in terms of $u$ - and $w$-coordinates is given by $u_{i, k}=\phi\left(w_{i, k}\right)$. To argue existence of (3.5) or equivalently (3.6) we need the following lemma whose proof is inspired by [21, Lemma 2.1]. We introduce the mapping

$$
\psi: \ell^{q / p} \rightarrow \ell^{q}, \text { by }(\psi(z))_{k}=\left|z_{k}\right|^{1 / p} \operatorname{sgn} z_{k}, \text { for } k=1, \ldots
$$

for scalar valued sequences $z$.

Lemma 3.2. Let $q>p$, and let $\beta$ denote the conjugate of $q / p$. Then the mapping $\psi: \ell^{q / p} \rightarrow l^{\beta}$ is weakly (sequentially) continuous, i.e. $z^{n} \rightarrow \bar{z}$ weakly in $\ell^{q / p}$ implies that $\psi\left(z^{n}\right) \rightarrow \psi(\bar{z})$ weakly in $\ell^{\beta}$.

Proof. First note that $\ell^{q} \subset \ell^{\beta}$, since $\beta=\frac{q}{q-p}>q$. Let $r=\frac{1}{p}+1$ and let $r^{*}$ denote the conjugate exponent of $r$ given by $r^{*}=p+1$. Then

$$
1<\frac{q}{p} \leq \frac{1}{p}<r
$$

which implies $r^{*}<\beta$. For any $z \in \ell^{q / p}$, we have

$$
\|z\|_{r}^{r}=\sum_{k=1}^{\infty}\left|z_{k}\right|^{r},\|\psi(z)\|_{r^{*}}^{r^{*}}=\sum_{k=1}^{\infty}\left|z_{k}\right|^{r^{*} / p}=\sum_{k=1}^{\infty}\left|z_{k}\right|^{r}
$$

and

$$
(\psi(z), z)_{\ell^{r^{*}, \ell^{r}}}=\sum_{k=1}^{\infty} \psi(z)_{k} \cdot z_{k}=\sum_{k=1}^{\infty}\left|z_{k}\right|^{1 / p+1}=\sum_{k=1}^{\infty}\left|z_{k}\right|^{r}
$$


The above computations imply that

$$
(\psi(z), z)_{\ell^{*}, \ell^{r}}=\|\psi(z)\|_{r^{*}}\|z\|_{r}, \text { and }\|\psi(z)\|_{r^{*}}^{r^{*}}=\|z\|_{r}^{r},
$$

which means that $\psi$ is the duality mapping from $\ell^{r}$ to $\ell^{r^{*}}$ and is weakly sequentially continuous. If $z^{n} \rightarrow \bar{z}$ weakly in $\ell^{q / p}$, then $z^{n} \rightarrow \bar{z}$ weakly in $\ell^{r}$ since $1<q / p<r$. Therefore, $\psi\left(z^{n}\right) \rightarrow \psi(\bar{z})$ weakly in $\ell^{r^{*}}$. Using that $r^{*}<\beta$, this implies that $\psi\left(z^{n}\right) \rightarrow \psi(\bar{z})$ weakly in $\ell^{\beta}$.

Theorem 3.3. There exists a minimizer $\bar{w}$ to $(3.6)$, and hence a minimizer $\bar{u}$ to 3.5 .

Proof. The case $p=q$ has been dealt with in [23]. Therefore, we focus on the case $q>p$.

Let $\left\{w^{n}=\left(w_{1}^{n}, \ldots, w_{m}^{n}\right)\right\}_{n=1}^{\infty} \subset \ell^{q / p}\left(\ell^{1}\right)$ denote a minimizing sequence for 3.6. We set $\left\{u^{n}=\left(u_{1}^{n}, \ldots, u_{m}^{n}\right)\right\}_{n=1}^{\infty} \subset \ell_{b}^{q / p}\left(\ell^{p}\right)$, where

$$
u_{i, k}^{n}=\psi\left(w_{i, k}^{n}\right)=b_{k}^{-1 / q}\left(\psi\left(w_{i}^{n}\right)\right)_{k}=b_{k}^{-1 / q}\left|w_{i, k}^{n}\right|^{1 / p} \operatorname{sgn}\left(w_{i, k}^{n}\right) \text {, for } i=1, \ldots, m, k=1, \ldots, n=1, \ldots
$$

Since $\left\{w^{n}\right\}$ is a minimizing sequence there exists a constant $K>0$ independent of $n$, such that

$$
\sum_{k=0}^{\infty}\left|w_{i, k}^{n}\right|^{q / p} \leq \sum_{k=0}^{\infty}\left(\sum_{i=1}^{m}\left|w_{i, k}^{n}\right|\right)^{q / p} \leq K
$$

for each $i=1, \ldots, m$. This implies that the scalar-valued sequences $\left\{w_{i, k}^{n}\right\}_{k=1}^{\infty}$ are bounded in $\ell^{q / p}$ uniformly with respect to $i=1, \ldots, m$ and $n=1, \ldots$. Hence there exists, for each $i$, a subsequence (denoted by the same symbols) and some $\bar{w}_{i} \in \ell^{q / p}$, such that $w_{i}^{n} \rightarrow \bar{w}_{i}$ in $\ell^{q / p}$, see e.g. [10. pp. 73]. From Lemma 3.2 we have that $\psi\left(w_{i}^{n}\right) \rightarrow\left(\bar{w}_{i}\right)$ weakly in $\ell^{\beta}$.

Let $y_{n}$ be the solution to 3.7) with control $w^{n}$. Then on each interval $I_{k}$, we can deduce by the Arzelà-Ascoli theorem that there exists $\bar{y}_{k}: I_{k} \rightarrow \mathbb{R}^{d}$ such that

$$
y_{n} \rightarrow \bar{y}_{k} \text { uniformly w.r.t. } t \in I_{k} \text {, as } n \rightarrow \infty .
$$

For $\bar{y}:[0, \infty) \rightarrow \mathbb{R}^{d}$ defined by $\left.\bar{y}\right|_{I_{k}}=\bar{y}_{k}$ for $k \in \mathbb{N}$, it follows that for any $T>0$

$$
y_{n} \rightarrow \bar{y} \text { uniformly in }[0, T) \text {, as } n \rightarrow \infty .
$$

Therefore, $\bar{y}$ is the solution to 3.7p corresponding to $\bar{w}:=\left(\bar{w}_{1}, \ldots, \bar{w}_{m}\right)$. Here we use that the dynamics $f$ is affine in $\psi\left(w_{i}\right), i=1, \ldots, m$. Using the fact that $y_{n} \rightarrow \bar{y}$ pointwise in $[0, \infty)$ and $w_{i, k}^{n} \rightarrow \bar{w}_{i, k}$ for any $i=1, \ldots, m, k \in \mathbb{N}$, we obtain by Fatou's lemma that

$$
\begin{gathered}
\int_{0}^{\infty} e^{-\lambda t} \frac{1}{2}\left\|\bar{y}(t)-y_{d}\right\|_{2}^{2} d t+\sum_{k=0}^{\infty}\left(\sum_{i=1}^{m}\left|\bar{w}_{i, k}\right|\right)^{q / p} \\
\leq \liminf _{n \rightarrow \infty} \int_{0}^{\infty} e^{-\lambda t} \frac{1}{2}\left\|y_{n}(t)-y_{d}\right\|_{2}^{2} d t+\sum_{k=0}^{\infty}\left(\sum_{i=1}^{m}\left|w_{i, k}^{n}\right|\right)^{q / p},
\end{gathered}
$$

which implies that $\bar{w}$ is a minimizer for problem (3.6), once we argue its admissability. For this purpose we observe that the weak convergence of $\psi\left(w_{i}^{n}\right)$ implies the strong convergence of $\psi\left(w_{i}^{n}\right)_{k} \rightarrow$ $\psi\left(\bar{w}_{i}\right)_{k}$ for each $k$. Since $\left(b_{k}^{-q} \psi\left(w_{1}^{n}\right)_{k}, \ldots, b_{k}^{-q} \psi\left(w_{m}^{n}\right)_{k}\right) \in U$ for each $k$ this implies that $\left(b_{k}^{-q} \psi\left(\bar{w}_{1}\right)_{k}, \ldots, b_{k}^{-q} \psi\left(\bar{w}_{m}\right)_{k}\right) \in U$ and thus $\bar{w}$ is admissible. 


\section{Sparsity and switching properties: the time-continuous prob- lem}

For the time-continuous problem (2.4), the Pontryagin maximum principle for infinite horizon problems has been widely studied the literature, see e.g. [4, 17, Theorem 3.2] and further references provided there. We have the following result.

Lemma 4.1. Assume that the discount factor $\lambda$ is sufficiently large. Then for each $x \in \mathbb{R}^{d}$, if $\bar{u}$ is a locally optimal control for problem 2.4) and $\bar{y}$ is the associated optimal trajectory, then there exists an adjoint state $\varphi:\left[0, \infty\left[\rightarrow \mathbb{R}^{d}\right.\right.$ such that

$$
-\dot{\varphi}(t)=D f_{0}(\bar{y}(t))^{*} \varphi(t)+\sum_{i=1}^{m} D f_{i}(\bar{y}(t))^{*} \bar{u}_{i}(t)+e^{-\lambda t}\left(\bar{y}(t)-y_{d}\right) \text {, a.e. } t>0,
$$

$\lim _{t \rightarrow \infty} \varphi(t)=0$, and for $\left.t \in\right] 0, \infty[$ a.e. we have

$$
\begin{aligned}
& \left\langle f_{0}(\bar{y}(t))+\sum_{i=1}^{m} f_{i}(\bar{y}(t)) \bar{u}_{i}(t), \varphi(t)\right\rangle+e^{-\lambda t}\left(\frac{1}{2}\left\|\bar{y}(t)-y_{d}\right\|_{2}^{2}+\gamma\|\bar{u}(t)\|_{p}^{q}\right) \\
\leq & \left\langle f_{0}(\bar{y}(t))+\sum_{i=1}^{m} f_{i}(\bar{y}(t)) u_{i}, \varphi(t)\right\rangle+e^{-\lambda t}\left(\frac{1}{2}\left\|\bar{y}(t)-y_{d}\right\|_{2}^{2}+\gamma\|u\|_{p}^{q}\right)
\end{aligned}
$$

for all $u \in U$.

Proof. We sketch a proof, verifying the assumptions of [4, Theorem 3.2, Corollary B.5]. It will be convenient to introduce $f(y, u)=f_{0}(y(t))+\sum_{i=1}^{m} f_{i}(y(t)) u_{i}(t)$ for the right hand side of (2.2). Condition (A1) of the cited result holds due to the structure $f$ and the assumption that the mappings $f_{i}$ are in $C^{1}\left(\mathbb{R}^{d}, \mathbb{R}^{d}\right)$. Since by assumption $(H 1)$ the set $U$ is compact the constant $M=s u p_{u \in U)}\|u\|_{2}$ is well-defined. Consequently by $(\mathrm{H} 2)$ we find that $v=L(1+m M)$ is a global Lipschitz constant for $f(\cdot, u)$, uniformly with respect to $u \in U$. By [6, Theorem III.5.5], we have that $\bar{y}(t) \leq \tilde{M} \sqrt{t} e^{v t}$ for some constant $\tilde{M}$ independent of $t \in[0, \infty)$. Therefore for any $\varepsilon>0$, there exists a constant $M_{\varepsilon}$ such that $\bar{y}(t) \leq M_{\varepsilon} e^{(v+\varepsilon) t}$. Then for $\lambda>2 v$ all assumptions of [4, Corollary B.5] are satisfied and we can conclude the existence of an adjoint state $\varphi$ satisfying (4.9).

Throughout the remainder of this section we assume that $\lambda>2 v$, with $v$ defined in the previous proof. We point out that the property $\lim _{t \rightarrow \infty} \varphi(t)=0$, will not be needed in this section. Suggested by (4.9), we shall investigate the minimizers of the following function

$$
G_{t}(u):=\sum_{i=1}^{m}\left\langle f_{i}(\bar{y}(t)), \varphi(t)\right\rangle u_{i}+\gamma e^{-\lambda t}\|u\|_{p}^{q}, \forall u \in U
$$

where $t \in] 0, \infty[$. We now assume that the set of control constraints $U$ has the form of box constraints:

$$
U_{\infty}:=\left\{u=\left(u_{1}, \ldots, u_{m}\right) \in \mathbb{R}^{m}:-\rho_{i} \leq u_{i} \leq \rho_{i}, i=1, \ldots, m\right\},
$$

where $\rho_{i}>0$. In this case the optimality condition can be used to derive the following structural properties of a minimizer.

Theorem 4.2. Let $\bar{u}$ be an optimal control for problem $(2.4)$ with $U_{\infty}$ given in $(4.10)$, let $\bar{y}$ be the associated optimal trajectory and $\varphi$ the associated adjoint state. For $t \in] 0, \infty[$ a.e., we define the following index sets:

$$
I^{-}(t)=\left\{i \in\{1, \ldots, m\}:\left|\left\langle f_{i}(\bar{y}(t)), \varphi(t)\right\rangle\right| \rho_{i}^{1-q}<\gamma e^{-\lambda t}\right\}
$$




$$
\begin{aligned}
& I^{0}(t)=\left\{i \in\{1, \ldots, m\}:\left|\left\langle f_{i}(\bar{y}(t)), \varphi(t)\right\rangle\right| \rho_{i}^{1-q}=\gamma e^{-\lambda t}\right\}, \\
& I^{+}(t)=\left\{i \in\{1, \ldots, m\}:\left|\left\langle f_{i}(\bar{y}(t)), \varphi(t)\right\rangle\right| \rho_{i}^{1-q}>\gamma e^{-\lambda t}\right\},
\end{aligned}
$$

Then the following properties hold:

(i) For $t \in] 0, \infty\left[\right.$ a.e. and $i \in I^{-}(t)$,

$$
\bar{u}_{i}(t)=0 .
$$

(ii) For $t \in] 0, \infty\left[\right.$ a.e. and $i \in I^{0}(t)$,

$$
\begin{cases}\bar{u}_{i}(t)=0, & \text { if } I^{+}(t) \neq \emptyset, \\ \bar{u}_{i}(t) \in\left\{0,-\rho_{i} \operatorname{sgn}\left(\left\langle f_{i}(\bar{y}(t)), \varphi(t)\right\rangle\right)\right\}, & \\ \bar{u}_{i}(t) \bar{u}_{j}(t)=0, i, j \in I^{0}(t), i \neq j, & \text { if } I^{+}(t)=\emptyset, q \in[p, 1[, \\ \bar{u}_{i}(t) \in\left[0,-\rho_{i} \operatorname{sgn}\left(\left\langle f_{i}(\bar{y}(t)), \varphi(t)\right\rangle\right)\right], & \\ \bar{u}_{i}(t) \bar{u}_{j}(t)=0, i, j \in I^{0}(t), i \neq j, & \text { if } I^{+}(t)=\emptyset, q=1 .\end{cases}
$$

(iii) For $t \in] 0, \infty\left[\right.$ a.e. and $i \in I^{+}(t)$, we have

$$
\bar{u}_{i}(t) \in\left\{0,-\rho_{i} \operatorname{sgn}\left(\left\langle f_{i}(\bar{y}(t)), \varphi(t)\right\rangle\right)\right\},
$$

with $\max _{i \in I^{+}(t)}\left|\bar{u}_{i}(t)\right| \neq 0$.

Let us briefly comment on sparsity and switching properties which follow from Theorem 4.2 For the coordinates in the index set $I^{-}(t)$, the controllers are zero. We refer to these coordinates as the sparse control coordinates at the time $t$. If $I^{+}(t)=\emptyset$, then $i \in I^{0}(t) \cup I^{-}(t)$ for all $i=1, \ldots, m$, and hence $u$ is switching or sparse at time $t$. If $I^{+}(t) \neq \emptyset$ then the coordinates in $I^{0}(t)$ behave like those in $I^{-}(t)$, they are 0 . The coordinates of the optimal control in the index set $I^{+}(t)$ are not completely determined by (iii). They are either active, or zero and thus they join the set of sparse control coordinates. Comparing to the case $p=q$ which was treated in [23, Proposition 5.2], the case (iii) is such that the control is necessarily active. Thus $p<q$ enhances additional sparsity compared to $p=q$. Finally, as a consequence of the box constraints, the optimal control is of bang-off-bang type, except for case (ii) with $q=1$.

Proof. We shall use that by Lemma 4.1 we know that $\bar{u}(t)$ minimizes $G_{t}$ in $U_{\infty}$ for a.e. $t \in(0, \infty)$. For convenience of notations, let us set

$$
\varphi_{t, i}=\left\langle f_{i}(\bar{y}(t)), \varphi(t)\right\rangle, \gamma_{t}=\gamma e^{-\lambda t} .
$$

In Step 1 below we verify (i) and (ii). The claims in (iii) are proved in Step 2.

Step 1: proof of (i) and (ii).

At first, let us focus on the case $q>p$. Consider further the case $\varphi_{t, i} \leq 0$ for $i=1, \ldots, m$. Then $\bar{u}_{i}(t) \geq 0$ for $i=1, \ldots, m$. We introduce

$$
\Omega:=\left\{u \in U_{\infty}: 0 \leq u_{i} \leq \rho_{i}, i=1, \ldots, m\right\} .
$$

Let us decompose $G_{t}$ in $\Omega$ as follows:

$$
G_{t}(u)=G_{1}(u)+\gamma_{t} G_{2}(u),
$$

where

$$
G_{1}(u)=\sum_{i=1}^{m} \varphi_{t, i} u_{i}+\gamma_{t} \sum_{i=1}^{m} u_{i}^{q}, G_{2}(u)=\left(\sum_{i=1}^{m} u_{i}^{p}\right)^{q / p}-\sum_{i=1}^{m} u_{i}^{q} .
$$


$G_{1}$ is a concave function in $\Omega, G_{2} \geq 0$, and $G_{2}=0$ if and only if $\sum_{i, j=1, \ldots, m, i \neq j}\left|u_{i} u_{j}\right|=0$. Here we use that $\frac{q}{p} \in\left[1, \frac{1}{p}\right]$ and the fact that

$$
\left(\sum_{i=1}^{m} a_{i}\right)^{r} \geq \sum_{i=1}^{m} a_{i}^{r}
$$

for each $a_{i} \geq 0, r>1$, and equality holds if and only if $a_{i} a_{j}=0$ for all $i, j=1, \ldots, m, i \neq j$. Then we deduce that

$$
G_{t}(u) \geq G_{1}(u),
$$

and equality holds if and only if $\sum_{i, j=1, \ldots, m, i \neq j}\left|u_{i} u_{j}\right|=0$.

If $I^{0}(t)=\emptyset$ and $I^{+}(t)=\emptyset$, i.e. $\rho^{1-q}\left|\varphi_{t, i}\right|<\gamma_{t}$ for $i=1, \ldots, m$, we have

$$
\left.\left.\varphi_{t, i} u_{i}+\gamma_{t} u_{i}^{q}>0 \text { for } u_{i} \in\right] 0, \rho_{i}\right],
$$

where $u=u(t)$. Therefore $G_{1}$ attains its unique minimum at $(0, \ldots, 0)$ and $G_{2}(0, \ldots, 0)=0$. Consequently $\bar{u}_{i}(t)=0$ for $i=1, \ldots, m$.

If $I^{+}(t)=\emptyset$, we have

$$
\left.\left.\varphi_{t_{i}} u_{i}+\gamma_{t} u_{i}^{q}>0 \text { for } u_{i} \in\right] 0, \rho\right], i \in I^{-}(t), \text { and } \varphi_{t, j} u_{j}+\gamma_{t} u_{j}^{q} \geq 0 \text { for } u_{j} \in\left[0, \rho_{j}\right], j \in I^{0}(t) .
$$

Moreover for $j \in I^{0}(t)$, the expression $\varphi_{t, j} u_{j}+\gamma_{t} u_{j}^{q}$ attains its minimum in $\left[0, \rho_{j}\right]$ at 0 and $\rho_{j}$ if $q<1$, and $\varphi_{t, j} u_{j}+\gamma_{t} u_{j}^{q} \equiv 0$ if $q=1$. Therefore,

$$
\bar{u}_{i}(t)=0 \text { for } i \in I^{-}(t), \bar{u}_{j}(t) \in\left\{0, \rho_{j}\right\} \text { for } j \in I^{0}(t), q<1, \text { and } \sum_{j, j^{\prime} \in I^{0}(t), j \neq j^{\prime}}\left|u_{j} u_{j^{\prime}}\right|=0,
$$

and

$$
\bar{u}_{i}(t)=0 \text { for } i \in I^{-}(t), \bar{u}_{j}(t) \in\left[0, \rho_{j}\right] \text { for } j \in I^{0}(t), q=1 \text {, and } \sum_{j, j^{\prime} \in I^{0}(t), j \neq j^{\prime}}\left|u_{j} u_{j^{\prime}}\right|=0 .
$$

If $I^{+}(t) \neq \emptyset$, we have

$$
\varphi_{t, i} u_{i}+\gamma u_{i}^{q} \geq 0 \text { for } u_{i} \in\left[0, \rho_{i}\right], i \in I^{-}(t) \cup I^{0}(t),
$$

and $\varphi_{t, j} u_{j}+\gamma u_{j}^{q}$ attains its unique minimum in $\left[0, \rho_{j}\right]$ at $\rho_{j}$ for $j \in I^{+}(t)$. Thus, for any $u \in \Omega$, we define $\tilde{u} \in \Omega$ as follows:

$$
\tilde{u}_{i}=0 \text { for } i \in I^{-}(t) \cup I^{0}(t) \text {, and } \tilde{u}_{i}=u_{i} \text { for } i \in I^{+}(t) .
$$

If $\tilde{u}=(0, \ldots, 0)$, then for any $j \in I^{+}(t)$ we set $\hat{u} \in \Omega$ with

$$
\hat{u}_{j}=\rho \text { and } \hat{u}_{i}=0 \text { for } i \neq j, i=1, \ldots, m .
$$

Thus we have

$$
G(u) \geq G_{1}(u) \geq G_{1}(\tilde{u})>G_{1}(\hat{u})=G(\hat{u}) .
$$

Otherwise if $\tilde{u} \neq(0, \ldots, 0)$ and $u \neq \tilde{u}$,

$$
G(u)=G_{1}(u)+\gamma_{t} G_{2}(u)>G_{1}(u)+\gamma_{t} G_{2}(\tilde{u}) \geq G_{1}(\tilde{u})+\gamma_{t} G_{2}(\tilde{u})=G(\tilde{u}) .
$$

We then deduce that

$$
\bar{u}_{i}(t)=0 \text { for } i \in I^{-}(t) \cup I^{0}(t) .
$$


The proof for the case when $\varphi_{t, i} \leq 0$ for $i=1, \ldots, m$ is thus concluded. The other cases when $\varphi_{t, i}$ have different signs can be treated analogously.

Now we proceed to look at the case $q=p$. In this situation, $G_{2} \equiv 0$ and $G \equiv G_{1}$. The minimizers of $G_{1}$ have been analyzed in the previous arguments, and we therefore arrive at the conclusion.

\section{Step 2: proof of (iii).}

We turn to analyze the behavior of the coordinates with indices in $I^{+}(t)$. In particular in this case $I^{+}(t) \neq \emptyset$, and consequently by (i) and (ii)

$$
\bar{u}_{i}(t)=0 \text {, for } i \in I^{-}(t) \cup I^{0}(t) .
$$

Therefore,

$$
G_{t}(\bar{u}(t))=\sum_{\tau=1}^{\ell} \varphi_{t, i_{\tau}} \bar{u}_{i_{\tau}}(t)+\gamma_{t}\left(\sum_{\tau=1}^{\ell}\left|\bar{u}_{i_{\tau}}(t)\right|^{p}\right)^{q / p},
$$

where $\left\{i_{1}, \ldots, i_{\ell}\right\} \subset\{1, \ldots, m\}$ is such that $I^{+}(t)=\left\{i_{1}, \ldots, i_{\ell}\right\}$. Then the problem consists in finding the minimizer of the function

$$
\tilde{G}(w):=\sum_{\tau=1}^{\ell} \psi_{\tau} w_{\tau}+\gamma_{t}\left(\sum_{\tau=1}^{\ell} \rho_{\tau}^{p}\left|w_{\tau}\right|^{p}\right)^{q / p}, \text { for } w=\left(w_{1}, \ldots, w_{\ell}\right) \in[-1,1]^{\ell},
$$

where, to simplify notation, we set for $\tau=1, \ldots, \ell$

$$
w_{\tau}=\frac{u_{i_{\tau}}}{\rho_{i_{\tau}}}, \psi_{\tau}=\varphi_{t, i_{\tau}} \rho_{i_{\tau}}, \text { and } \rho_{\tau}=\rho_{i_{\tau}}
$$

Following the definition of $I^{+}(t)$, we have

$$
\left|\psi_{\tau}\right| \rho_{\tau}^{-q}>\gamma_{t}, \text { for } \tau=1, \ldots, \ell .
$$

Let $\bar{w}$ be the minimizer and let us start by considering the case

$$
\psi_{\tau}<0, \text { for all } \tau=1, \ldots, \ell
$$

Then it is trivial to see that

$$
\bar{w}_{\tau} \geq 0, \text { for all } \tau=1, \ldots, \ell .
$$

We aim to prove that the minimizer $\bar{w}$ is not in the interior of $[0,1]^{\ell}$. Without loss of generality, we assume that

$$
1 \geq \bar{w}_{1} \geq \bar{w}_{2} \geq \cdots \geq \bar{w}_{\ell} \geq 0 .
$$

We can therefore limit our attention to the subset

$$
\left\{\left(w_{1}, \ldots, w_{\ell}\right): 1 \geq w_{1} \geq w_{2} \geq \cdots w_{\ell} \geq 0\right\} .
$$

Note that $\bar{w}$ can be expressed as $\bar{w}=\left(\beta_{0} \bar{w}_{1}, \beta_{1} \beta_{0} \bar{w}_{1}, \ldots, \beta_{\ell-1} \ldots \beta_{0} \bar{w}_{1}\right)$ where $\beta_{0}=1$, and $\beta_{\tau} \in$ $[0,1], \tau=1, \ldots, \ell-1$. Moreover $\bar{w}_{1} \in[0,1]$ is a minimizer of the functional

$$
G_{\beta, 1}\left(w_{1}\right)=\sum_{\tau=1}^{\ell} \psi_{\tau} \beta_{\tau-1} \cdots \beta_{0} w_{1}+\gamma_{t}\left(\sum_{\tau=1}^{\ell} \rho_{\tau}^{p} \beta_{\tau-1}^{p} \cdots \beta_{0}^{p}\right)^{q / p} w_{1}^{q}
$$

We will exclude the case that $w_{1} \rightarrow G_{\beta, 1}\left(w_{1}\right)$ assumes a minimum in the interior of $[0,1]$. 
Indeed, if such a minimum $w_{1}^{*}$ is attained in the interior of $[0,1]$, then

$$
0=G_{\beta, 1}^{\prime}\left(w_{1}^{*}\right)=\sum_{\tau=1}^{\ell} \psi_{\tau} \beta_{\tau-1} \cdots \beta_{0}+\gamma_{t}\left(\sum_{\tau=1}^{\ell} \rho_{\tau}^{p} \beta_{\tau-1}^{p} \cdots \beta_{0}^{p}\right)^{q / p} q\left(w_{1}^{*}\right)^{q-1} .
$$

Therefore,

$$
G_{\beta, 1}\left(w_{1}^{*}\right)=(1-q) \gamma_{t}\left(\sum_{\tau=1}^{\ell} \rho_{\tau}^{p} \beta_{\tau-1}^{p} \cdots \beta_{0}^{p}\right)^{q / p} q\left(w_{1}^{*}\right)^{q} \geq 0
$$

Note that

$$
G_{\beta, 1}(1,0, \ldots, 0)=\psi_{1}+\gamma_{t} \rho_{1}^{q}=\rho_{1}^{q}\left(-\left|\psi_{1}\right| \rho_{1}^{-q}+\gamma_{t}\right)<0,
$$

where (4.14) is applied. Thus,

$$
G_{\beta, 1}\left(w_{1}^{*}\right)>G_{\beta, 1}(1,0, \ldots, 0),
$$

which contradicts the assumption that $w_{1}^{*}$ is the minimizer. Consequently, the minimum can not be attained in the interior of $[0,1]$ and thus $\bar{w}_{1} \in\{0,1\}$. Moreover $G_{\beta, 1}(1,0, \ldots, 0)<0$ and $G_{\beta, 1}(0, \ldots, 0)=$ 0 , and thus

$$
\bar{w}_{1}=1 .
$$

We next claim the following: for $j \in\{2, \ldots, \ell-1\}$, if $\bar{w}_{j-1} \in\{0,1\}$, then

$$
\bar{w}_{j} \in\{0,1\},
$$

and verify this statement by induction. If $\bar{w}_{j-1}=0$, by 4.15 we have

$$
\bar{w}_{j}=0
$$

as claimed. If $\bar{w}_{j-1}=1$, then $\bar{w}_{\tau}=1$, for all $\tau=1, \ldots, j-1$.

To characterize further $\bar{w}_{j}$, we apply the same idea as for determining $\bar{w}_{1}$. This time we restrict our attention to the subset

$$
\left\{\left(w_{j}, w_{j+1}, \ldots, w_{\ell}\right): 1 \geq w_{j} \geq w_{j+1} \geq \cdots \geq w_{\ell} \geq 0\right\},
$$

and note that for the optimal $\left(\bar{w}_{j}, \ldots, \bar{w}_{\ell}\right)=\left(\bar{w}_{j}, \beta_{j} \bar{w}_{j}, \ldots, \beta_{\ell-1} \ldots \beta_{j} \bar{w}_{j}\right)$, where $\beta_{\tau} \in[0,1]$ for $\tau=$ $1, \ldots, \ell-1$. We denote for any $w_{j} \in[0,1]$

$$
\begin{aligned}
G_{\beta, j}\left(w_{j}\right)= & \sum_{\tau=1}^{j-1} \psi_{\tau}+\psi_{j} w_{j}+\sum_{\tau=j+1}^{\ell} \psi_{\tau} \beta_{\tau-1} \cdots \beta_{j} w_{j} \\
& +\gamma_{t}\left[\sum_{\tau=1}^{j-1} \rho_{\tau}^{p}+\rho_{j}^{p} w_{j}^{p}+\sum_{\tau=j+1}^{\ell} \rho_{\tau}^{p} \beta_{\tau-1}^{p} \cdots \beta_{j}^{p} w_{j}^{p}\right]^{q / p},
\end{aligned}
$$

and note that $\bar{w}_{j}$ is a minimizer of $G_{\beta, j}$ on $[0,1]$. If a minimum $w_{j}^{*}$ is attained in the interior of $[0,1]$, then

$$
G_{\beta, j}^{\prime}\left(w_{j}^{*}\right)=0 .
$$

This yields that,

$$
\psi_{j}+\sum_{\tau=j+1}^{\ell} \psi_{\tau} \beta_{\tau-1} \cdots \beta_{j}+\gamma_{t} \frac{q}{p} S_{j}^{q / p-1}\left(\rho_{j}^{p}+\sum_{\tau=j+1}^{\ell} \rho_{\tau}^{p} \beta_{\tau-1}^{p} \cdots \beta_{j}^{p}\right) p\left(w_{j}^{*}\right)^{p-1}=0,
$$


where

$$
S_{j}=\sum_{\tau=1}^{j-1} \rho_{\tau}^{p}+\rho_{j}^{p}\left(w_{j}^{*}\right)^{p}+\sum_{\tau=j+1}^{\ell} \rho_{\tau}^{p} \beta_{\tau-1}^{p} \cdots \beta_{j}^{p}\left(w_{j}^{*}\right)^{p}
$$

Therefore,

$$
\psi_{j}+\sum_{\tau=j+1}^{\ell} \psi_{\tau} \beta_{\tau-1} \cdots \beta_{j}=-\gamma_{t} S_{j}^{q / p-1}\left(\rho_{j}^{p}+\sum_{\tau=j+1}^{\ell} \rho_{\tau}^{p} \beta_{\tau-1}^{p} \cdots \beta_{j}^{p}\right) q\left(w_{j}^{*}\right)^{p-1} .
$$

By applying the above equality to compute $G_{\beta, j}\left(w_{j}^{*}\right)$, we obtain

$$
\begin{aligned}
& G_{\beta, j}\left(w_{j}^{*}\right) \\
= & \sum_{\tau=1}^{j-1} \psi_{\tau}+\left(\psi_{j}+\sum_{\tau=j+1}^{\ell} \psi_{\tau} \beta_{\tau-1} \cdots \beta_{j}\right) w_{j}^{*}+\gamma_{t} S_{j}^{q / p} \\
= & \sum_{\tau=1}^{j-1} \psi_{\tau}-\gamma_{t} S_{j}^{q / p-1}\left(\rho_{j}^{p}+\sum_{\tau=j+1}^{\ell} \rho_{\tau}^{p} \beta_{\tau-1}^{p} \cdots \beta_{j}^{p}\right) q\left(w_{j}^{*}\right)^{p}+\gamma_{t} S_{j}^{q / p} \\
= & \sum_{\tau=1}^{j-1} \psi_{\tau}+\gamma_{t} S_{j}^{q / p-1}\left[\sum_{\tau=1}^{j-1} \rho_{\tau}^{p}+(1-q)\left(\rho_{j}^{p}+\sum_{\tau=j+1}^{\ell} \rho_{\tau}^{p} \beta_{\tau-1}^{p} \cdots \beta_{j}^{p}\right)\left(w_{j}^{*}\right)^{p}\right] .
\end{aligned}
$$

Using the fact that $q \leq 1$ and $w_{j}^{*}>0$, it holds that

$$
\begin{aligned}
G_{\beta, j}\left(w_{j}^{*}\right) & \geq \sum_{\tau=1}^{j-1} \psi_{\tau}+\gamma_{t} S_{j}^{q / p-1} \sum_{\tau=1}^{j-1} \rho_{\tau}^{p} \\
& >\sum_{\tau=1}^{j-1} \psi_{\tau}+\gamma_{t}\left(\sum_{\tau=1}^{j-1} \rho_{\tau}^{p}\right)^{q / p-1} \sum_{\tau=1}^{j-1} \rho_{\tau}^{p} \\
& =\sum_{\tau=1}^{j-1} \psi_{\tau}+\gamma_{t}\left(\sum_{\tau=1}^{j-1} \rho_{\tau}^{p}\right)^{q / p} \\
& =G_{\beta, j}(0),
\end{aligned}
$$

which contradicts the assumption that $w_{j}^{*}$ is the minimizer. Consequently, the minimum can not be attained in the interior of $[0,1]$. We then deduce that

$$
\bar{w}_{j} \in\{0,1\},
$$

which completes the proof for the claim 4.17). Together with 4.16, it is deduced that

$$
\bar{w}_{\tau} \in\{0,1\}, \text { for } \tau=1, \ldots, \ell,
$$

which concludes the case where $\psi_{\tau}<0$ for all $\tau=1, \ldots, \ell$.

For the other cases where $\psi_{\tau}$ is positive for some $\tau \in\{1, \ldots, \ell\}, \psi_{\tau}$ and $w_{\tau}$ can be replaced by $-\psi_{\tau}$ and $-w_{\tau}$ in 4.12 . Then by following the same arguments as in the previously we can obtain that $-\bar{w}_{\tau} \in\{0,1\}$. Therefore we conclude that

$$
\bar{w}_{\tau} \in\left\{0,-\operatorname{sgn}\left(\psi_{\tau}\right)\right\}, \text { for } \tau=1, \ldots, \ell,
$$

with the additional information that $\left|\bar{w}_{1}\right|=1$. The definition of $w_{\tau}$ and $\psi_{\tau}$ in 4.13 implies that

$$
\bar{u}_{i_{\tau}} \in\left\{0,-\rho_{i_{\tau}} \operatorname{sgn}\left(\varphi_{t, i_{\tau}}\right)\right\}, \text { for } i_{\tau} \in I^{+}(t), \tau=1, \ldots, \ell
$$

with the additional information that $\max _{i \in I^{+}} \frac{\left|\bar{u}_{i}\right|}{\rho_{i}} \neq 0$. This completes the proof of (iii). 
In Theorem 4.2 the study has been made for the case of box constraints. Next we briefly consider the problem under Euclidean norm constraints. In this case, due to the coupling of the coordinates which is inherent to the Euclidean norm, it appears to be more complicated to achieve explicit information on the structure of the minimizers compared to that which was obtained for box constraints.

We define for $\rho>0$

$$
U_{2}:=\left\{u=\left(u_{1}, \ldots, u_{m}\right) \in \mathbb{R}^{m}: \sum_{i=1}^{m} u_{i}^{2} \leq \rho^{2}\right\} .
$$

Theorem 4.3. Let $\bar{u}$ be an optimal control for problem (2.4) with $U$ given in (4.18), let $\bar{y}$ be the associated optimal trajectory, and $\varphi$ its associated adjoint state. Let $I^{-}(t), I^{0}(t)$ and $I^{+}(t)$ be as defined in Theorem 4.2 If for some $t \in] 0, T$ [ the cardinality of $I^{+}(t)$ is less or equal to 1 , then $(i)$, (ii), and (iii) of that theorem remain valid. Otherwise we have

$$
\left.\sum_{i=1}^{m}\left|\bar{u}_{i}(t)\right|^{2}=\rho^{2}, \text { for a.e. } t \in\right] 0, \infty[\text {. }
$$

Proof. Step 1. From 4.9] we know that for $t \in] 0, \infty[$ a.e., $\bar{u}(t)$ is the minimizer of the following function

$$
\bar{G}_{t}(u):=\sum_{i=1}^{m} \alpha_{i}(t) u_{i}+\gamma e^{-\lambda t}\|u\|_{p}^{q}, \text { for all } u \in U_{2},
$$

where $\alpha_{i}(t)=\left\langle f_{i}(\bar{y}(t)), \varphi(t)\right\rangle$. At first we note that $U_{2}$ is a subset of $U_{\infty}$, if $\rho_{i}=\rho$ for all $i$, and hence $\min _{u \in U_{\infty}} \bar{G}_{t}(u) \leq \min _{u \in U_{2}} \bar{G}_{t}(u)$. Moreover, if a minimizer of $\bar{G}_{t}$ over $U_{\infty}$ is contained in $U_{2}$, then this minimizer is also a minimizer of $\bar{G}_{t}$ over $U_{2}$. Following this observation, let $\bar{u}(t)$ be a minimizer of $\bar{G}_{t}$ over $U_{\infty}$ with cardinality of $I^{+}(t) \leq 1$. Then by Theorem 4.2 all components of $\bar{u}(t)$ are 0 except for at most one. In case the cardinality of $I^{+}(t)$ equals one, then there is one non-trivial coordinate of the control at time $t$ whose norm then equals $\rho$.

Step 2. Now we turn to the general case (assuming that $I^{+}$is nonempty) and prove that the optimal control is necessarily active. Since $I^{+}(t)$ is non-empty there exists at least one index $\tau$ such that $\gamma_{t}-\left|\alpha_{\tau}(t)\right| \rho^{1-q}<0$. Setting the value of this coordinate equal to $\rho$ we obtain

$$
G((0, \ldots 0, \rho, 0, \ldots, 0))=\alpha_{\tau}(t) \rho+\gamma_{t} \rho^{q}=\rho^{q}\left(\gamma_{t}+\alpha_{\tau}(t) \rho^{1-q}\right)=\rho^{q}\left(\gamma_{t}-\left|\alpha_{\tau}(t)\right| \rho^{1-q}\right)<0,
$$

which implies that at least one coordinate of $\bar{u}$ is nontrivial and $G(\bar{u}(t))<0$. Let $\tilde{\ell}$ denote the number of nontrivial coordinates of $\bar{u}$ and without loss of generality assume that these are the $\tilde{\ell}$ first ones of $\bar{u}(t)$.

Let us start with the case where $\alpha_{i}(t) \leq 0$ for all $i=1, \ldots, \tilde{\ell}$. It is trivial to see that $\bar{u}_{i}(t) \geq 0$, for $i=1, \ldots, \tilde{\ell}$ in this case. We set

$$
\Omega:=\left\{\left(u_{1}, \ldots, u_{\tilde{\ell}}\right) \in \mathbb{R}^{\tilde{\ell}}: \sum_{i=1}^{\tilde{\ell}} u_{i}^{2} \leq \rho^{2}, u_{i} \geq 0, i=1, \ldots, \tilde{\ell}\right\} .
$$

Thus $\bar{u}(t) \in \Omega$. We prove by contradiction that $\bar{u} \in \partial \Omega$. If this is not the case, i.e. $\bar{u}$ is in the interior of $\Omega$, then

$$
\frac{\partial G}{\partial u_{i}}(\bar{u}(t))=0, i=1, \ldots, \tilde{\ell}
$$

from which we deduce that

$$
\alpha_{i}(t)+\gamma_{t} \frac{q}{p}\left(\sum_{j=1}^{\tilde{\ell}}\left|\bar{u}_{j}(t)\right|^{p}\right)^{q / p-1} p\left|\bar{u}_{i}(t)\right|^{p-1}=0, i=1, \ldots, \tilde{\ell}
$$


It follows that

$$
\alpha_{i}(t) \bar{u}_{i}(t)=-\gamma_{t} q\left(\sum_{j=1}^{\tilde{\ell}}\left|\bar{u}_{j}(t)\right|^{p}\right)^{q / p-1}\left|\bar{u}_{i}(t)\right|^{p}=0, i=1, \ldots, \tilde{\ell}
$$

Therefore,

$$
\begin{aligned}
G(\bar{u}(t)) & =\sum_{i=1}^{\tilde{\ell}} \alpha_{i}(t) \bar{u}_{i}(t)+\gamma_{t}\left(\sum_{i=1}^{\tilde{\ell}}\left|\bar{u}_{i}(t)\right|^{p}\right)^{q / p} \\
& =-\gamma_{t} q\left(\sum_{i=1}^{\tilde{\ell}}\left|\bar{u}_{i}(t)\right|^{p}\right)^{q / p-1}\left|\bar{u}_{i}(t)\right|^{p}+\gamma_{t}\left(\sum_{i=1}^{\tilde{\ell}}\left|\bar{u}_{i}(t)\right|^{p}\right)^{q / p} \\
& =\gamma_{t}(1-q)\left(\sum_{i=1}^{\tilde{\ell}}\left|\bar{u}_{i}(t)\right|^{p}\right)^{q / p} \geq 0 .
\end{aligned}
$$

Since we already know that $G(\bar{u}(t))<0$ this gives a contradiction. Consequently $\bar{u}(t) \in \partial \Omega$. Since $\bar{u}_{i} \neq 0$ for $i=1, \ldots, \tilde{\ell}$ this implies that

$$
\sum_{i=1}^{\tilde{\ell}} \bar{u}_{i}^{2}(t)=\rho^{2} \text { and } \bar{u}_{i}(t)>0, \text { for } i=1, \ldots, \tilde{\ell} .
$$

If some of the coordinates of $\alpha$ are such that $\alpha_{i}(t) \geq 0$, then necessarily $\bar{u}_{i}(t) \leq 0$ and, adapting $\Omega$ accordingly, it can again be verified that $\sum_{1}^{l} \bar{u}_{i}^{2}(t)=\bar{\rho}^{2}$.

\section{Sparsity and switching properties: the time-discretized prob- lem}

In this subsection we consider the following linear dynamical system: for $x \in \mathbb{R}^{d}$,

$$
\left\{\begin{array}{l}
\dot{y}(t)=A y(t)+B u(t) \\
y(0)=x
\end{array}\right.
$$

where $A \in \mathbb{R}^{d \times d}$ and $B \in \mathbb{R}^{d \times m}$. Let us recall the optimal control problem: given $x \in \mathbb{R}^{d}$, consider

$$
\inf \left\{J^{\Delta}(x, u):(y, u) \text { satisfies } 5.20, u \in U^{\Delta}\right\} .
$$

The cost functional is recalled as follows:

$$
J^{\Delta}(x, u)=\int_{0}^{\infty} \frac{1}{2} e^{-\lambda t}\left\|y(t)-y_{d}\right\|_{2}^{2} d t+\gamma R(u),
$$

where

$$
R(u)=\sum_{k=0}^{\infty} b_{k}\left(\sum_{i=1}^{m}\left|u_{i, k}\right|^{p}\right)^{q / p} \text { for } u \in U^{\Delta} .
$$

To investigate the optimality conditions satisfied by the optimal controllers, we introduce firstly the adjoint equation associated to $(y, u)$ satisfying [5.20):

$$
\left\{\begin{array}{l}
-\dot{\varphi}(t)=A^{T} \varphi(t)+e^{-\lambda t}\left(y(t)-y_{d}\right) \quad \text { for } t>0 \\
\lim _{t \rightarrow \infty} \varphi(t)=0
\end{array}\right.
$$


In the remainder of this section we assume that there exists an adjoint state $\varphi$ satisfying (5.22). The following result justifies this assumption. We denote by $\sigma(A)$ the set of eigenvalues of $A$, and $\sigma:=\sup \{\operatorname{Re} \mu: \mu \in \sigma(A)\}$. Further we define

$$
L_{\lambda}^{2}\left(0, \infty ; \mathbb{R}^{d}\right):=\left\{y \in L^{2}\left(0, \infty ; \mathbb{R}^{d}\right)\right\}: e^{\left.\lambda \cdot y \in L^{2}\left(0, \infty ; \mathbb{R}^{d}\right)\right\}}
$$

with $\|y\|_{L_{\lambda}^{2}\left(0, \infty ; \mathbb{R}^{d}\right)}^{2}=\int_{0}^{\infty} e^{\lambda t}|y(t)|^{2} d t$.

Lemma 5.1. If $\lambda>2 \max \{0, \sigma\}$, then 5.22 admits a unique solution $\varphi \in L_{\lambda}^{2}\left(0, \infty ; \mathbb{R}^{d}\right)$ with $\dot{\varphi} \in$ $L_{\lambda}^{2}\left(0, \infty ; \mathbb{R}^{d}\right)$.

Proof. If $y$ is feasible for 5.21, and in particular if it is optimal, then as a consequence of the cost functional and the inhomogeneity in 5.22) it follows that

$$
e^{-\lambda t}\left(y(t)-y_{d}\right) \in L_{\lambda}^{2}\left(0, \infty ; \mathbb{R}^{d}\right)
$$

This suggests to investigate, for arbitrary $g \in L_{\lambda}^{2}\left(0, \infty ; \mathbb{R}^{d}\right)$, the following equation has a solution:

$$
\left\{\begin{array}{l}
-\dot{\varphi}(t)=A^{T} \varphi(t)+g(t), \text { a.e. } t>0, \\
\lim _{t \rightarrow \infty} \varphi(t)=0 .
\end{array}\right.
$$

We define

$$
W_{\lambda}^{1,2}:=\left\{y \in L_{\lambda}^{2}\left(0, \infty ; \mathbb{R}^{d}\right): \dot{y} \in L_{\lambda}^{2}\left(0, \infty ; \mathbb{R}^{d}\right)\right\},
$$

endowed with $\|y\|_{W_{\lambda}^{1,2}}^{2}=\|y\|_{L_{\lambda}^{2}\left(0, \infty ; \mathbb{R}^{d}\right)}^{2}+\|\dot{y}\|_{L_{\lambda}^{2}\left(0, \infty ; \mathbb{R}^{d}\right)}^{2}$ as norm. Note that $y \in W_{\lambda}^{1,2}$ implies that $t \mapsto y$ is continuous on $[0, \infty)$. Hence $W_{\lambda, 0}^{1,2}=\left\{y \in W_{\lambda}^{1,2}: y(0)=0\right\}$ is well defined. For any $\varphi \in W_{\lambda, 0}^{1,2}$ we have $e^{\frac{\lambda}{2}} \varphi \in L^{2}\left(0, \infty ; \mathbb{R}^{d}\right)$ and $\frac{d}{d t}\left(e^{\frac{\lambda \cdot}{2}} \varphi\right) \in L^{2}\left(0, \infty ; \mathbb{R}^{d}\right)$. This implies that $\lim _{t \rightarrow \infty} e^{\frac{\lambda t}{2}} \varphi(t)=0$ and hence $\lim _{t \in \infty} \varphi(t)=0$.

By the definition of $\sigma$ there exists a constant $M>0$ such that

$$
\left\|e^{A t} y\right\|_{2} \leq M e^{\sigma t}\|y\|_{2}, \text { for all } t \in[0, \infty) \text { and } y \in \mathbb{R}^{d} .
$$

We now define the bounded linear operator $T: W_{\lambda, 0}^{1,2} \rightarrow L_{\lambda}^{2}\left(0, \infty ; \mathbb{R}^{d}\right)$ by

$$
T y=\dot{y}-A y+\lambda y \text {. }
$$

The adjoint operator $T^{*}: L_{\lambda}^{2}\left(0, \infty ; \mathbb{R}^{d}\right) \rightarrow\left(W_{\lambda, 0}^{1,2}\right)^{*}$ satisfies

$$
\begin{aligned}
\left\langle T^{*} \varphi, y\right\rangle_{\left(W_{\lambda, 0}^{1,2}\right)^{*}, W_{\lambda, 0}^{1,2}} & =\langle\varphi, \dot{y}\rangle_{L_{\lambda}^{2}\left(0, \infty ; \mathbb{R}^{d}\right)}-\langle\varphi, A y-\lambda y\rangle_{L_{\lambda}^{2}\left(0, \infty ; \mathbb{R}^{d}\right)} \\
& =-\langle\dot{\varphi}, y\rangle_{\left(W_{\lambda, 0}^{1,2}\right)^{*}, W_{\lambda, 0}^{1,2}}-\langle\lambda \varphi, y\rangle_{L_{\lambda}^{2}\left(0, \infty ; \mathbb{R}^{d}\right)}-\left\langle A^{T} \varphi-\lambda \varphi, y\right\rangle_{L_{\lambda}^{2}\left(0, \infty ; \mathbb{R}^{d}\right)} \\
& =-\langle\dot{\varphi}, y\rangle_{\left(W_{\lambda, 0}^{1,2}\right)^{*}, W_{\lambda, 0}^{1,2}}-\left\langle A^{T} \varphi, y\right\rangle_{L_{\lambda}^{2}\left(0, \infty ; \mathbb{R}^{d}\right)},
\end{aligned}
$$

where we use that $y(0)=0$ and $\lim _{t \rightarrow \infty} e^{\frac{\lambda t}{2}} y(t)=0$ in the second equality above. Thus $T^{*} \varphi=$ $-\dot{\varphi}-A^{T} \varphi$ in the variational sense.

Let us next argue that $T$ is surjective. We choose $f \in L_{\lambda}^{2}\left(0, \infty ; \mathbb{R}^{d}\right)$ arbitrarily and define

$$
y(t)=\int_{0}^{t} e^{(A-\lambda I)(t-s)} f(s) d s .
$$


Then we have

$$
\dot{y}=A y-\lambda y+f
$$

and

$$
\begin{aligned}
\|y\|_{L_{\lambda}^{2}\left(0, \infty ; \mathbb{R}^{d}\right)} & =\left[\int_{0}^{\infty} e^{\lambda t}\left|\int_{0}^{t} e^{(A-\lambda I)(t-s)} f(s) d s\right|^{2} d t\right]^{1 / 2} \\
& \leq M\left[\int_{0}^{\infty} e^{\lambda t}\left(\int_{0}^{t} e^{(\sigma-\lambda)(t-s)}|f(s)| d s\right)^{2} d t\right]^{1 / 2} \\
& =M\left[\int_{0}^{\infty}\left(\int_{0}^{t} e^{\left(\sigma-\frac{\lambda}{2}\right)(t-s)} e^{\frac{\lambda s}{2}}|f(s)| d s\right)^{2} d t\right]^{1 / 2},
\end{aligned}
$$

and by the Young's inequality

$$
\|y\|_{L^{2}\left(0, \infty ; \mathbb{R}^{d}\right)} \leq M \int_{0}^{\infty} e^{\left(\sigma-\frac{\lambda}{2}\right)} d t\|f\|_{L_{\lambda}^{2}\left(0, \infty ; \mathbb{R}^{d}\right)}=\frac{2 M}{\lambda-2 \sigma}\|f\|_{L_{\lambda}^{2}\left(0, \infty ; \mathbb{R}^{d}\right)} .
$$

Moreover, by (5.24)

$$
\|\dot{y}\|_{L^{2}\left(0, \infty ; \mathbb{R}^{d}\right)} \leq\left[\frac{2 M}{\lambda-2 \sigma}(\|A\|+\lambda)+1\right]\|f\|_{L_{\lambda}^{2}\left(0, \infty ; \mathbb{R}^{d}\right)}
$$

and hence $y \in W_{\lambda, 0}^{1,2}$ as desired. Thus $T$ is surjective and is clearly injective, and the same holds for $T^{*}$. By the closed range theorem there exists a constant $C>0$ such that

$$
\|\varphi\|_{L_{\lambda}^{2}\left(0, \infty ; \mathbb{R}^{d}\right)} \leq C\left\|T^{*} \varphi\right\|_{\left(W_{\lambda, 0}^{1,2}\right)} \text { for all } \varphi \in L_{\lambda}^{2}\left(0, \infty ; \mathbb{R}^{d}\right) .
$$

Let $g \in L_{\lambda}^{2}\left(0, \infty ; \mathbb{R}^{d}\right)$ be arbitrary and choose $\varphi \in L_{\lambda}^{2}\left(0, \infty ; \mathbb{R}^{d}\right)$ such that $T^{*} \varphi=g$. Then by 5.25

$$
\|\varphi\|_{L_{\lambda}^{2}\left(0, \infty ; \mathbb{R}^{d}\right)} \leq C\|g\|_{\left(W_{\lambda, 0}^{1,2}\right)} \leq C \tilde{C}\|g\|_{L_{\lambda}^{2}\left(0, \infty ; \mathbb{R}^{d}\right)}
$$

where $\tilde{C}$ is the embedding constant of $L_{\lambda}^{2}\left(0, \infty ; \mathbb{R}^{d}\right)$ into $\left(W_{\lambda, 0}^{1,2}\right)^{*}$. Moreover $\dot{\varphi}=-A^{T} \varphi-g$ and thus

$$
\|\dot{\varphi}\|_{L_{\lambda}^{2}\left(0, \infty ; \mathbb{R}^{d}\right)} \leq(C \tilde{C}+1)\|g\|_{L_{\lambda}^{2}\left(0, \infty ; \mathbb{R}^{d}\right)} .
$$

It follows that $\varphi \in W_{\lambda}^{1,2}$ and $\lim _{t \rightarrow \infty} \varphi(t)=0$. Thus $\varphi$ is the solution to 5.23 and the claim of the lemma follows.

Since the controls in $U^{\Delta}$ are piecewise constant functions, we consider at first the optimal control on each time interval $I_{k}, k \in \mathbb{N}$.

Proposition 5.2. Assume that $\lambda>\max \{0,2 \sigma\}$. Let $\tilde{u} \in U^{\Delta}$ satisfy the following: for any $k \in \mathbb{N}$, $\tilde{u}(\cdot) \equiv \tilde{u}_{k}$ in $I_{k}$ and

$$
\tilde{u}_{k} \in \underset{u=\left(u_{1}, \ldots, u_{m}\right) \in U}{\arg \min }\left\{\int_{I_{k}}\langle\tilde{\varphi}(t), B u\rangle d t+\gamma b_{k}\|u\|_{p}^{q}\right\},
$$

where $\tilde{y}$ is the corresponding trajectory and $\tilde{\varphi}$ is the adjoint state associated to $(\tilde{y}, \tilde{u})$. Further for any arbitrary $\omega \in \mathbb{R}^{m}$ such that $\omega+\tilde{u}_{k} \in U$, we define the perturbed control

$$
u^{\omega}(t):= \begin{cases}\tilde{u}_{k}+\omega & \text { if } t \in I_{k}, \\ \tilde{u}(t) & \text { otherwise. }\end{cases}
$$

Then it holds that $J^{\Delta}\left(x, u^{\omega}\right) \geq J^{\Delta}(x, \tilde{u})$. 
Proof. Let $y^{\omega}$ be the trajectory associated with $u^{\omega}$. Then $y^{\omega}-\tilde{y}$ satisfies

$$
\left\{\begin{array}{l}
\dot{y}^{\omega}(t)-\dot{\tilde{y}}(t)=A\left(y^{\omega}(t)-y(t)\right)+B \omega \mathbb{1}_{I_{k}}(t) \quad \text { for } t \in(0, \infty), \\
y^{\omega}(0)-\tilde{y}(0)=0 .
\end{array}\right.
$$

Assumption (5.26) implies that

$$
\int_{I_{k}}\left\langle\tilde{\varphi}(t), B\left(\tilde{u}_{k}+\omega\right)\right\rangle d t+\gamma b_{k}\left\|\tilde{u}_{k}+\omega\right\|_{p}^{q} \geq \int_{I_{k}}\left\langle\tilde{\varphi}(t), B \tilde{u}_{k}\right\rangle d t+\gamma b_{k}\left\|\tilde{u}_{k}\right\|_{p}^{q} .
$$

By the definition of $u^{\omega}$, 5.27) is equivalent to

$$
\int_{I_{k}}\langle\tilde{\varphi}(t), B \omega\rangle d t+\gamma R\left(u^{\omega}\right)-\gamma R(\tilde{u}) \geq 0 .
$$

For almost all $t>0$ we obtain,

$$
\begin{aligned}
& \frac{d}{d t}\left\langle\tilde{\varphi}(t), y^{\omega}(t)-\tilde{y}(t)\right\rangle \\
= & \left\langle\tilde{\tilde{\varphi}}(t), y^{\omega}(t)-\tilde{y}(t)\right\rangle+\left\langle\tilde{\varphi}(t), \dot{y}^{\omega}(t)-\tilde{\tilde{y}}(t)\right\rangle \\
= & \left\langle-A^{T} \tilde{\varphi}(t)-e^{-\lambda t}\left(\tilde{y}(t)-y_{d}\right), y^{\omega}(t)-\tilde{y}(t)\right\rangle+\left\langle\tilde{\varphi}(t), A\left(y^{\omega}(t)-\tilde{y}(t)\right)+B \omega \mathbb{1}_{I_{k}}(t)\right\rangle \\
= & -e^{-\lambda t}\left\langle\tilde{y}(t)-y_{d}, y^{\omega}(t)-\tilde{y}(t)\right\rangle+\left\langle\tilde{\varphi}(t), B \omega \mathbb{1}_{I_{k}}(t)\right\rangle .
\end{aligned}
$$

Note that $\lim _{t \rightarrow \infty} \tilde{\varphi}(t)=0$ and $y^{\omega}(0)-\tilde{y}(0)=0$, and therefore

$$
\int_{0}^{\infty} \frac{d}{d t}\left\langle\tilde{\varphi}(t), y^{\omega}(t)-\tilde{y}(t)\right\rangle=0
$$

Consequently we obtain

$$
\int_{0}^{\infty}\left[-e^{-\lambda t}\left\langle\tilde{y}(t)-y_{d}, y^{\omega}(t)-\tilde{y}(t)\right\rangle+\left\langle\tilde{\varphi}(t), B \omega \mathbb{1}_{l_{k}}(t)\right\rangle\right] d t=0,
$$

i.e.,

$$
\int_{0}^{\infty} e^{-\lambda t}\left\langle\tilde{y}(t)-y_{d}, y^{\omega}(t)-\tilde{y}(t)\right\rangle d t=\int_{I_{k}}\langle\tilde{\varphi}(t), B \omega\rangle .
$$

To compute the left-hand side of (5.29), we have for every $t>0$

$$
\left\|y^{\omega}(t)-y_{d}\right\|_{2}^{2}-\left\|\tilde{y}(t)-y_{d}\right\|_{2}^{2}=\left\|y^{\omega}(t)-\tilde{y}(t)\right\|_{2}^{2}+2\left\langle y^{\omega}(t)-\tilde{y}(t), \tilde{y}(t)-y_{d}\right\rangle,
$$

and now (5.28), 5.29) and (5.30) imply that

$$
\begin{aligned}
& \int_{0}^{\infty} \frac{1}{2} e^{-\lambda t}\left\|y^{\omega}(t)-y_{d}\right\|_{2}^{2} d t-\int_{0}^{\infty} \frac{1}{2} e^{-\lambda t}\left\|\tilde{y}(t)-y_{d}\right\|_{2}^{2} d t \\
& -\int_{0}^{\infty} \frac{1}{2} e^{-\lambda t}\left\|y^{\omega}(t)-\tilde{y}(t)\right\|_{2}^{2} d t+\gamma R\left(u^{\omega}\right)-\gamma R(\tilde{u}) \geq 0 .
\end{aligned}
$$

Then we deduce that

$$
J^{\Delta}\left(x, u^{\omega}\right)-J^{\Delta}(x, \tilde{u}) \geq \int_{0}^{\infty} \frac{1}{2} e^{-\lambda t}\left\|y^{\omega}(t)-\tilde{y}(t)\right\|_{2}^{2} d t \geq 0,
$$

which ends the proof.

Proposition 5.2 provides the way to construct optimal controls on each $I_{k}$, and this procedure can be naturally extended to construct globally optimal controls. 
Theorem 5.3. Assume that $\lambda>\max \{0,2 \sigma\}$. Let $\bar{u} \in U^{\Delta}$ satisfy the following: for any $k \in \mathbb{N}$ and $t \in I_{k}$,

$$
\bar{u}(t) \in \underset{u=\left(u_{1}, \ldots, u_{m}\right) \in U}{\operatorname{argmin}}\left\{\int_{I_{k}}\langle\bar{\varphi}(t), B u\rangle d t+\gamma b_{k}\|u\|_{p}^{q}\right\},
$$

where $\bar{y}$ is the corresponding trajectory and $\bar{\varphi}$ is the adjoint state associated to $(\bar{y}, \bar{u})$. Then $\bar{u} \in U^{\Delta}$ is a minimizer of problem 3.5 , i.e.

$$
J^{\Delta}(x, u) \geq J^{\Delta}(x, \bar{u}), \forall u \in U^{\Delta} .
$$

Proof. For any $u \in U^{\Delta}$, we define a sequence $\left(u^{n}\right)_{n \in \mathbb{N}}$ by

$$
u^{n}(t):= \begin{cases}\bar{u}(t) & \text { if } t \in I_{k}, k=0, \ldots, n \\ u(t) & \text { otherwise. }\end{cases}
$$

Therefore, $u^{n} \rightarrow \bar{u}$ pointwise in $\left[0, \infty\left[\right.\right.$. Let $y^{n}$ be the trajectory associated with $u^{n}$. By the same argument as in Theorem 3.3 , we deduce that

$$
y^{n} \rightarrow \bar{y} \text { pointwise in }[0, \infty[.
$$

Assumption 5.31 and Proposition 5.2 imply that

$$
J^{\Delta}(x, u) \geq J^{\Delta}\left(x, u^{0}\right) \geq J^{\Delta}\left(x, u^{1}\right) \geq \cdots \geq J^{\Delta}\left(x, u^{n}\right), \forall n \in \mathbb{N} .
$$

By Fatou's Lemma,

$$
\begin{aligned}
J^{\Delta}(x, u) & \geq \liminf _{n \rightarrow \infty}\left\{\int_{0}^{\infty} \frac{1}{2} e^{-\lambda t}\left\|y^{n}(t)-y_{d}\right\|_{2}^{2} d t+\gamma R\left(u^{n}\right)\right\} \\
& \geq \int_{0}^{\infty} \frac{1}{2} e^{-\lambda t}\left\|\bar{y}(t)-y_{d}\right\|_{2}^{2} d t+\gamma R(\bar{u}) \\
& =J^{\Delta}(x, \bar{u}),
\end{aligned}
$$

and we conclude that $\bar{u}$ is a minimizer of problem 3.5 .

Based on the optimality conditions 5.31, similar results on sparsity and switching properties as Theorem 4.2 can be deduced by the same arguments as in the proof of Theorem 4.2

Theorem 5.4. Following the same assumptions and notations in Theorem 5.3. we set

$$
\varphi_{k}=\int_{I_{k}} B^{T} \bar{\varphi}(t) d t, \gamma_{k}=\gamma b_{k} .
$$

For each $k \in \mathbb{N}$, we define the following index sets:

$$
\begin{aligned}
& I_{k}^{-}=\left\{i \in\{1, \ldots, m\}:\left|\varphi_{k, i}\right| \rho_{i}^{1-q}<\gamma_{k}\right\}, \\
& I_{k}^{0}=\left\{i \in\{1, \ldots, m\}:\left|\varphi_{k, i}\right| \rho_{i}^{1-q}=\gamma_{k}\right\}, \\
& I_{k}^{+}=\left\{i \in\{1, \ldots, m\}:\left|\varphi_{k, i}\right| \rho_{i}^{1-q}>\gamma_{k}\right\},
\end{aligned}
$$

The following properties hold:

(i) For $k \in \mathbb{N}$, $t \in I_{k}$ and $i \in I_{k}^{-}$,

$$
\bar{u}_{i}(t)=0 .
$$


(ii) For $k \in \mathbb{N}, t \in I_{k}$ and $i \in I_{k}^{0}$,

$$
\begin{cases}\bar{u}_{i}(t)=0, & \text { if } I_{k}^{+} \neq \emptyset, \\ \bar{u}_{i}(t) \in\left\{0,-\rho_{i} \operatorname{sgn}\left(\varphi_{k, i}\right)\right\}, & \\ \bar{u}_{i}(t) \bar{u}_{j}(t)=0, i, j \in I_{k}^{0}, i \neq j, & \text { if } I_{k}^{+}=\emptyset, q \in[p, 1[, \\ \bar{u}_{i}(t) \in\left[0,-\rho_{i} \operatorname{sgn}\left(\varphi_{k, i}\right)\right], & \\ \bar{u}_{i}(t) \bar{u}_{j}(t)=0, i, j \in I_{k}^{0}, i \neq j, & \text { if } I_{k}^{+}=\emptyset, q=1 .\end{cases}
$$

(iii) For $k \in \mathbb{N}, t \in I_{k}$ and $i \in I_{k}^{+}$, we have

$$
\bar{u}_{i}(t) \in\left\{0,-\rho_{i} \operatorname{sgn}\left(\varphi_{k, i}\right)\right\},
$$

and $\max _{i I_{k}^{+}}\left|\bar{u}_{i}(t)\right| \neq 0$.

\section{Numerical experiments}

In this section we present numerical experiments for the computation of optimal control laws for the problem

$$
\inf _{u(\cdot) \in L^{\infty}\left(0, \infty ; U_{\infty}\right)} J(x, u):=\int_{0}^{\infty} e^{-\lambda t}\left(\frac{1}{2}\left\|y(t)-y_{d}\right\|_{2}^{2}+\gamma\|u(t)\|_{p}^{q}\right) d t,
$$

constrained to the nonlinear dynamical system

$$
\left\{\begin{array}{l}
\left.\dot{y}(s)=f(y(s), u(s)):=f_{0}(y(s))+\sum_{i=1}^{m} f_{i}(y(s)) u_{i}(s) \quad \text { in }\right] 0, \infty[, \\
y(0)=x .
\end{array}\right.
$$

For the realization of globally optimal control laws we proceed as in [23], i.e. by following a dynamic programming approach. The value function $V(x):=\inf J(x, u)$ associated to this infinite horizon optimal control problem satisfies the following first order Hamilton-Jacobi-Bellman equation

$$
\lambda V(x)+\sup _{u \in U_{\infty}}\left\{-f(x, u) \cdot \nabla V(x)-\frac{1}{2}\left\|x-y_{d}\right\|_{2}^{2}-\gamma\|u\|_{p}^{q}\right\}=0,
$$

which leads to the optimal feedback map

$$
\bar{u}(x):=\underset{u \in U_{\infty}}{\operatorname{argmin}}\left\{f(x, u) \cdot \nabla V(x)+\gamma\|u\|_{p}^{q}\right\} .
$$

The solution of the Hamilton-Jacobi-Bellman equation and of the optimal feedback mapping are numerically approximated by a first-order semi-Lagrangian scheme with policy iteration as discussed in [2]. The well-posedness of this numerical scheme is guaranteed under boundedness and continuity assumptions for the dynamics $f(x, u)$ and the cost. Convergence of controls, however, is only guaranteed for convex running costs. Nevertheless, the results we report indicate that the semiLagrangian scheme converges to optimal controls exhibiting the expected sparsity and switching properties. This scheme has also been applied to the solution of sparse optimal feedback control problems in [1, 14]. In the case $p=q=1$ the minimization operation in (6.32) can be realized by means of semismooth Newton methods as [22]. For different values of $p$ and $q$, the minimizer is chosen by discretizing the control set $U_{\infty}$ into a finite number of values and making a pointwise evaluation of the Hamiltonian. 


\section{Eikonal dynamics}

We begin by considering eikonal-type dynamics for planar motion of the form

$$
\begin{aligned}
& \dot{x}_{1}(s)=u_{1}(s) \\
& \dot{x}_{2}(s)=u_{2}(s),
\end{aligned}
$$

where $\left|u_{i}(s)\right| \leq 0.5$ for $i=1,2$. The state space is set to be $\Omega=[-1,1]^{2}$, the discount factor $\lambda=0.2$, and $\gamma=1$. The goal is to drive the state to the origin, and therefore $y_{d}=(0,0)$. The optimal control fields in the state space for different $p, q$ values are shown in Figure 2

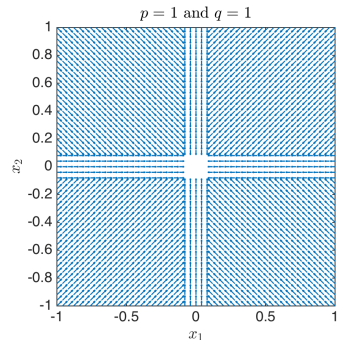

(a) $p=1, q=1$

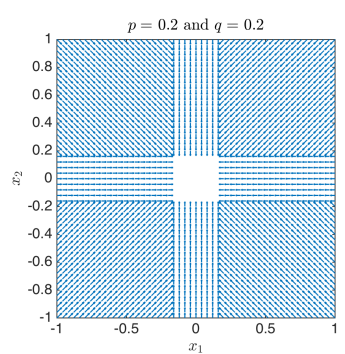

(d) $p=0.2, q=0.2$

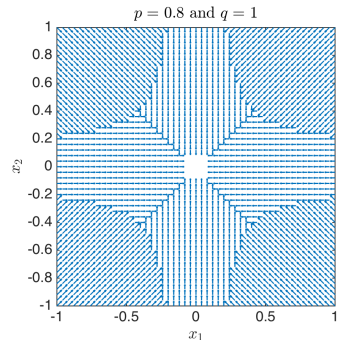

(b) $p=0.8, q=1$

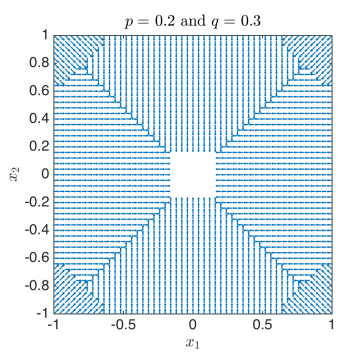

(e) $p=0.2, q=0.3$

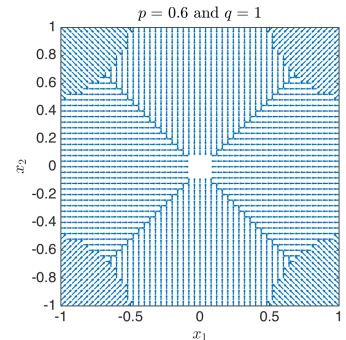

(c) $p=0.6, q=1$

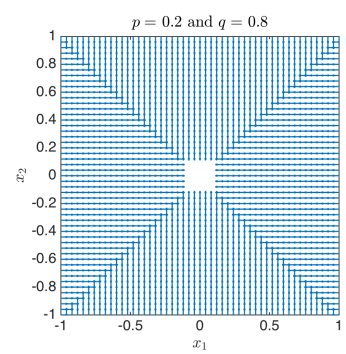

(f) $p=0.2, q=0.8$

Figure 2: Eikonal dynamics, optimal control fields for different control penalizations $\|u\|_{p}^{q}$.

We observe the following:

a) The case $p=q=1$ has been already reported in [23]. There exists a switching band of width $\gamma \lambda$, where the optimal control points unidirectionally towards the origin, and $\bar{u}=0$ for $\|u\|_{\infty} \leq \gamma \lambda$.

b,c) Departing from $p=q=1$ and reducing the value of $p$, a switching region with only one active control component arises. It increases as the ratio $q / p$ increases. Note that for $q=1$, the region where $\bar{u}=0$ remains unchanged.

d) The switching and the sparsity regions are larger for $p=q=0.2$ than for $p=q=1$. Only in the particular case $\rho=1$ these regions would remain the same.

e,f) Increasing the $q / p$ ratio by departing from smaller values of $q$ generates a larger switching region, leading to a fully switching controller for a ratio of $q / p$ sufficiently large. Note that increasing $q / p$ for $q \neq 1$ also leads to a decrease of the sparsity region. 


\section{Nonlinear dynamics of a double-well potential}

We now address the synthesis of optimal controllers for nonlinear dynamics. We consider a system corresponding to a single one-dimensional particle moving in a double-well potential, subject to a controlled damping, and a direct external forcing via

$$
\begin{aligned}
& \dot{x}(s)=v(s) \\
& \dot{v}(s)=-\left(1+u_{1}(s)\right) v(s)+\left(x(s)-x^{3}(s)\right)+u_{2}(s) .
\end{aligned}
$$

In the absence of control action $\left(u_{1}=u_{2}=0\right)$, the damped particle has two stable equilibrium positions, namely $x= \pm 1, v=0$ (we drop the state-space notation $\left(x_{1}, x_{2}\right)$ for $(x, v)$ ), with their corresponding basins of attraction. Here our goal is to steer the particle to the equilibrium $y_{d}=(1,0)$. We consider a set of initial conditions in $\Omega=[-2,2]^{2}$, and set $\gamma=0.1, \rho=1$, and $\lambda=0.01$. Optimal controls are shown in Figure 3 .

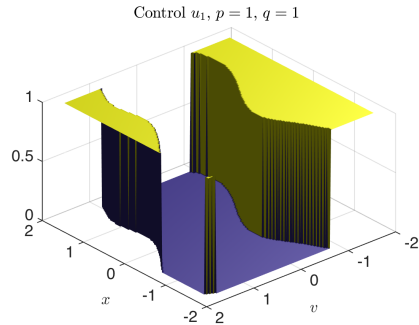

(a) $u_{1}, p=1, q=1$

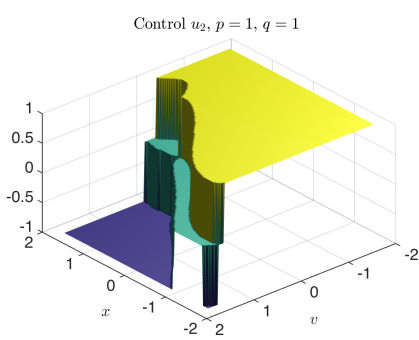

(d) $u_{2}, p=1, q=1$

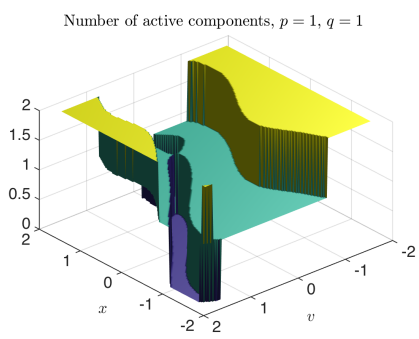

(g) $\|u\|_{0}, p=1, q=1$



(b) $u_{1}, p=0.6, q=1$

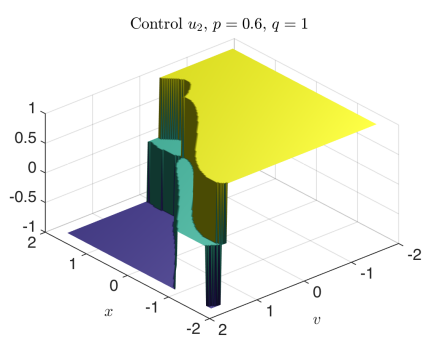

(e) $u_{2}, p=0.6, q=1$

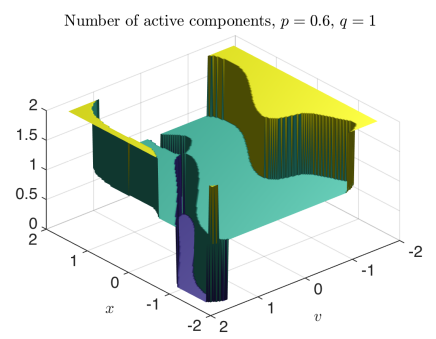

(h) $\|u\|_{0}, p=0.6, q=1$

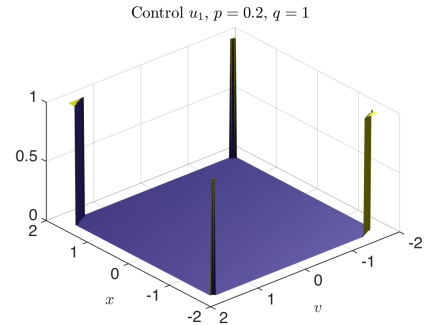

(c) $u_{1}, p=0.2, q=1$



(f) $u_{2}, p=0.2, q=1$

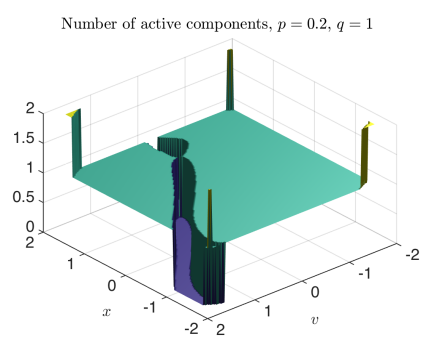

(i) $\|u\|_{0}, p=0.2, q=1$

Figure 3: Optimal controls for the double-well nonlinear control problem. The first two rows show the control variables $u_{1}$ and $u_{2}$ for different values of $p$ and $q$.

We observe: 
a,b,c) By reducing the value of $p$ with $q=1$, the region where the control $u_{1}$ is active decreases.

d,e,f) Reducing $q$ does not affect the sparsity pattern of $u_{2}$. The linear control action via $u_{2}$ is more relevant for the stabilization goal than the bilinear control term $u_{1} v$. As expected it becomes insignificant as $v$ becomes small.

$\mathrm{g}, \mathrm{h}, \mathrm{i})$ Overall, the reduction of $p$ has a significant effect on the increase of the switching region.

In order to investigate a setting with a richer interplay between the control variables and the switching structure, we consider a modified version of the double-well control system given by

$$
\begin{aligned}
& \dot{x}(s)=v(s) \\
& \dot{v}(s)=-\left(1+u_{1}(s)\right) v(s)+\left(x(s)-x^{3}(s)\right)+u_{2}(s) x(s),
\end{aligned}
$$

where $u_{2}$ enters now in a bilinear fashion. The optimal controllers are significantly different compared to the previous setting, as shown in Figure 4.

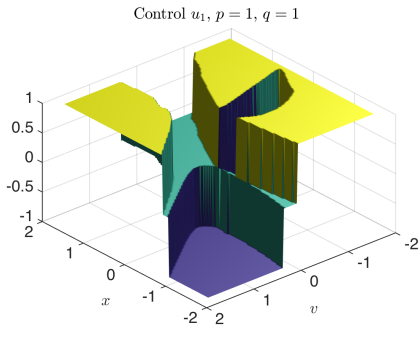

(a) $u_{1}, p=1, q=1$

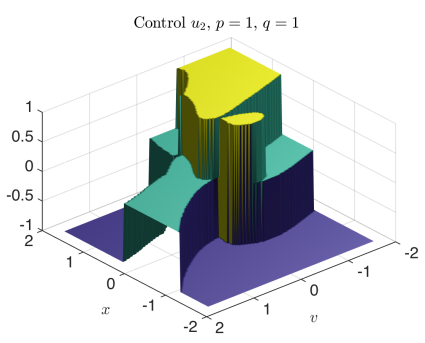

(d) $u_{2}, p=1, q=1$

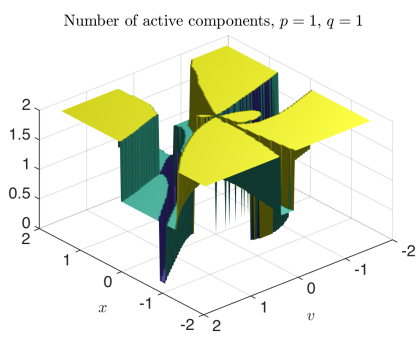

(g) $\|u\|_{0}, p=1, q=1$

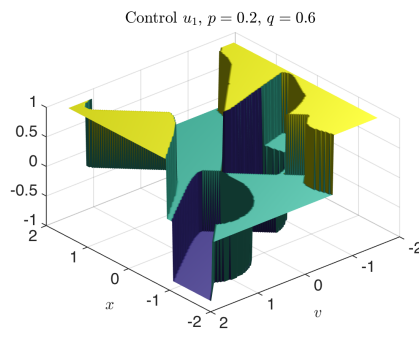

(b) $u_{1}, p=0.2, q=0.6$



(e) $u_{2}, p=0.2, q=0.6$

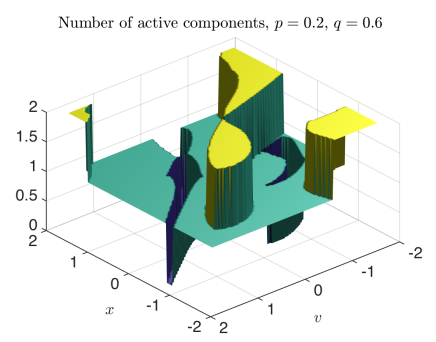

(h) $\|u\|_{0}, p=0.2, q=0.6$



(c) $u_{1}, p=0.2, q=1$

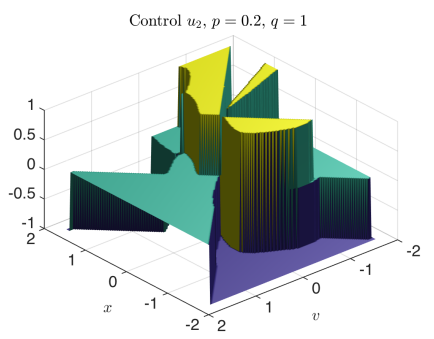

(f) $u_{2}, p=0.2, q=1$

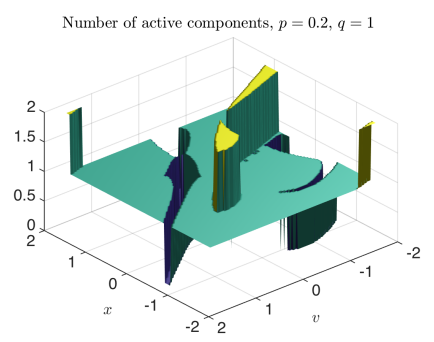

(i) $\|u\|_{0}, p=0.2, q=1$

Figure 4: Optimal controls for the double-well control problem with two bilinear controls. The first two rows show the control variables $u_{1}$ and $u_{2}$ for different values of $p$ and $q$. 
We note that:

a,b,c) The sparsity region of $u_{1}$ increases as the ratio $q / p$ increases.

$\mathrm{d}, \mathrm{e}, \mathrm{f})$ The sparsity region of $u_{2}$ also increases as $q / p$ increases.

$\mathrm{g}, \mathrm{h}, \mathrm{i})$ Overall, the switching pattern of the two control variables becomes dominant as the ratio $q / p$ becomes large. Only a reduced region of the state space requires the simultaneous action of two control variables.

Concluding remarks. In this paper we have studied infinite horizon optimal control problems with a control cost of the form $\|u\|_{p}^{q}$, where $0<p \leq q \leq 1$, leading to a non-convex, non-smooth optimization problem. From the analysis of the associated optimality conditions, we have shown that such control penalizations induce not only sparsity, but also a switching structure in the optimal control field. The switching pattern is determined by the different parameters of the control problem, but most notably, by the value of $q$ and the ratio $q / p$. By means of dynamic programming techniques, we have shown numerically that, for an increased $q / p$ ratio the optimal control has a dominant switching pattern, tending to minimize a counting $\|\cdot\|_{0}$ measure over an enlarged region of the state space. We believe that an important direction for future research is a thorough study of the interplay between the underlying dynamical structure of the control system and the switching pattern. More concretely, it would be desirable to know whether the sparse/switching control does benefit from the basin of attraction of a given equilibrium point, or whether the inclusion of $\|\cdot\|_{p}^{q}$ norms could lead to minimum time-type controllers.

\section{References}

[1] Albi, G., Fornasier, M. and Kalise, D.: A Boltzmann approach to mean-field sparse feedback control. IFAC-PapersOnLine 50(1)(2017):2898-2903.

[2] Alla, A., Falcone, M. and Kalise, D.: An efficient policy iteration algorithm for the solution of dynamic programming equations. SIAM J. Sci. Comput. 37(1)(2015):A181-A200.

[3] Alt, W. and Schneider, C.: Linear-quadratic control problems with $L_{1}$-control cost. Optim. Control Appl. Methods 36(2015):512-534.

[4] Aseev, S.M. and Veliov, V.M.: Another View of the Maximum Principle for Infinite-Horizon Optimal Control Problems in Economics, Research report 2017, ORCOS, Vienna University of Technology (2017).

[5] Aubin, J.P., and Cellina, A.: Differential Inclusions: Set-Valued Maps and Viability Theory. Grundlehren der Mathematischen Wissenschaften [Fundamental Principles of Mathematical Sciences], vol. 264., Springer-Verlag, Berlin (1984).

[6] Bardi, M., and Capuzzo-Dolcetta, I.: Optimal Control and Viscosity Solutions of HamiltonJacobi-Bellman Equations. Systems \& Control: Foundations \& Applications. Birkhäuser Boston, Inc., Boston, MA (1997).

[7] Bach, F., Jenatton, R., Mairal, J., and Obozinski, G.: Structured sparsity through convex optimization. Stat. Sci. 27(2012):450-468.

[8] Casas, E., Clason, C., and Kunisch, K.: Approximation of elliptic control problems in measure spaces with sparse solutions. SIAM J. Control Optim. 50(4)(2012):1735-1752. 
[9] Cannarsa, P., and Sinestrari, C.: Semiconcave Functions, Hamilton-Jacobi Equations, and Optimal Control. Progress in Nonlinear Differential Equations and their Applications, 58. Birkhäuser Boston, Inc., Boston, MA (2004)

[10] Cioranescu, I.: Geometry of Banach Spaces, Duality Mappings and Nonlinear Problems. Mathematics and Its Applications, Volume 62, Kluwer Academic Publishers, 1990.

[11] Clason, C., Ito, K. and Kunisch, K.: A convex analysis approach to optimal controls with switching structure for partial differential equations. ESAIM Control Optim. Calc. Var. 22(2)(2016):581-609.

[12] Clason, C., Kunisch, K., and Rund, A.: Nonconvex penalization of switching control of partial differential equations. preprint.

[13] Clason, C., Rund, A., Kunisch, K., and Barnard, R.: A convex penalty for switching control of partial differential equations. Systems Control Lett. 89(2016):66-73.

[14] Falcone, M., Kalise, D. and Kroener, A.: A semi-Lagrangian scheme for Lp-penalized minimum time problems. Proceedings of the 21st International Symposium on Mathematical Theory of Networks and Systems, 1798-1803 (2014).

[15] Fornasier, M. and Rauhut, H.: Recovery algorithms for vector-valued data with joint sparsity constraints. SIAM J. Numer. Anal. 46(2)(2008):577-613.

[16] Hájek, O.: $L_{1}$-Optimization in linear systems with bounded controls. J. Optim. Theory Appl. 29(3)(1979):409-436.

[17] Halkin, H.: Necessary conditions for optimal control problems with infinite horizons. Econometrica 42(2)(1974):267-272.

[18] Hante, F., Leugering, G., and Seidman, T.: Modeling and analysis of modal switching in networked transport system. Appl. Math. Optim. 59(2009):275-292.

[19] Hante, F., and Sager, S.: Relaxation methods for mixed-integer optimal control of partial differential equations. Comput. Optim. Appl. 55(2013):197-225.

[20] Herzog, R., Stadler, G., and Wachsmuth, G.: Directional sparsity in optimal control of partial differential equations. SIAM J. Control Optim. 50(2)(2012):943-963.

[21] Ito, K. and Kunisch, K.: A variational approach to sparsity optimization based on Lagrange multiplier theory. IOP Science Inverse Probl. 30(2014):015001.

[22] Kalise, D. , Kroener. A. and Kunisch, K.: Local minimization algorithms for dynamic programming equations. SIAM J. Sci. Comput. 38(3)(2016):A1587-A1615.

[23] Kalise, D., Kunisch, K., and Rao, Z.: Infinite horizon sparse optimal control. J. Optim. Theory Appl. 172(2)(2017):481-517.

[24] Kowalski, M.: Sparse regression using mixed norms. Appl. Comput. Harm. Anal. 27(2009):303-324.

[25] Pieper, K., and Vexler, B.: A priori error analysis for discretization of sparse elliptic optimal control problems in measure space. SIAM J. Control Optim. 51(4)(2013):2788-2808. 
[26] Teschke G., and Ramlau R., An iterative algorithm for nonlinear inverse problems with joint sparsity constraints in vector-valued regimes and an application to color image inpainting, Inverse Probl. 23(2007):1851-1870.

[27] Vinter, R.: Optimal Control. New York, NY, USA : Springer, 2010.

[28] Vossen, G., and Maurer, H.: On $L^{1}$-minimization in optimal control and applications to robotics. Optimal Control Appl. Methods 27(6)(2006):301-321.

[29] Wright, S., Nowak, R., and Figueiredo, M.: Sparse reconstruction by separable approximation. IEEE Trans. Signal Process. 57(2009):2479-2493.

[30] Yong, E.: Systems governed by ordinary differential equations with continuous, switching and impulse controls. Appl. Math. Optim. 20(1989):223-235.

[31] Zuazua, E.: Switching control. J. Eur. Math. Soc. 13(1)(2011):85-117.

[32] Zhao, P., Rocha, G., and Yu, B.: The composite absolute penalties family for grouped and hierarchical variable selection. Ann. Statist. 37(6A)(2009):3468-3497. 